\title{
An optical/NIR survey of globular clusters in early-type galaxies
}

\section{Ages of globular cluster systems and the relation to galaxy morphology}

\author{
A. L. Chies-Santos ${ }^{1}$, S. S. Larsen ${ }^{1}$, H. Kuntschner ${ }^{3}$, P. Anders ${ }^{1}$, E. M. Wehner ${ }^{1,2}$,
} J. Strader ${ }^{4}$, J. P. Brodie ${ }^{5}$, and J. F. C. Santos Jr. ${ }^{6}$

\author{
1 Astronomical Institute, University of Utrecht, Princetonplein 5, 3584 Utrecht, The Netherlands \\ e-mail: A.L.Chies@uu.nl \\ 2 Department of Physics and Astronomy, McMaster University, Hamilton L8S 4M1, Canada \\ 3 Space Telescope European Coordinating Facility, European Southern Observatory, Garching, Germany \\ ${ }^{4}$ Harvard-Smithsonian Center for Astrophysics, Cambridge, MA 02138, USA \\ 5 UCO/Lick Observatory, University of California, Santa Cruz, CA 95064, USA \\ ${ }^{6}$ Departamento de Física, ICEx, Universidade Federal de Minas Gerais, Av. Antônio Carlos 6627, Belo Horizonte 31270-901, MG, \\ Brazil
}

Received 2 September 2010 / Accepted 21 October 2010

\begin{abstract}
Context. Some photometric studies of extragalactic globular cluster (GC) systems using the optical and near-infrared colour combination have suggested the presence of a large fraction of intermediate-age (2-8 Gyr) GCs.

Aims. We investigate the age distributions of GC systems in $14 \mathrm{E} / \mathrm{S} 0$ galaxies.

Methods. We carried out a differential comparison of the $(g-z)$ vs. $(g-K)$ two-colour diagrams for GC systems in the different galaxies in order to see whether there are any indications of age differences. We also compared the different GC systems with a few simple stellar population models.

Results. No significant difference is detected in the mean ages of GCs among elliptical galaxies. S0 galaxies, on the other hand, show evidence for younger GCs. Surprisingly, this appears to be driven by the more metal-poor clusters. The age distribution of GCs in NGC 4365 seems to be similar to that of other large ellipticals (e.g. NGC 4486, NGC 4649). Padova SSPs with recently released isochrones for old ages $(14 \mathrm{Gyr})$ show less of an offset with respect to the photometry than previously published models.

Conclusions. We suggest that E type galaxies assembled most of their GCs in a shorter and earlier period than S0 type galaxies. The latter galaxy type seems to have a more extended period of GC formation/assembly.
\end{abstract}

Key words. galaxies: elliptical and lenticular, cD - galaxies: evolution - galaxies: star clusters: general

\section{Introduction}

Whenever a major star formation burst occurs, globular clusters (GCs) are expected to form in significant amounts (e.g. Ashman \& Zepf 1992; Elmegreen \& Efremov 1997; Forbes et al. 1997b; Brodie \& Strader 2006). In the $\Lambda$ CDM universe, examples of such events that shape the evolution of galaxies are infall of gas into single dark matter haloes and (gas rich) mergers. The latter are the main mechanism behind the hierarchical merging scenario (e.g. White 1978; Springel et al. 2005).

GCs still remain the best approximations of simple stellar populations (SSP), despite the recent findings of some clusters hosting multiple stellar populations (e.g. Piotto et al. 2007, in the Milky Way). It is difficult to resolve the individual stars of a galaxy beyond the Local Group. On the other hand, GCs can be easily detected at distances as far away as the Virgo and Fornax clusters. Hence, they have become a powerful tool in the study of galaxies in the local Universe. Observations of GCs translated into, for example, ages and metallicities are now widely used to put constraints on the formation and evolution galaxies (Brodie \& Strader 2006)

The importance of dating GCs stems from the possibility of retracing the major epochs of star formation in a galaxy. For extragalactic, unresolved GCs, only integrated properties can be measured. Optical colours suffer from the well known age/metallicity degeneracy (Worthey 1994). This degeneracy can in principle be lifted by combining optical and near-infrared (NIR) colours (e.g. Puzia et al. 2002; Hempel et al. 2003; Larsen et al. 2005). Generally studies using the optical/NIR colour combination have derived ages by mainly comparing GC colours with SSP models (Hempel et al. 2007a,b; Kotulla et al. 2008; Brodie \& Strader 2006, and references therein). The typical conclusion from these studies is that these galaxies mainly host old clusters (with ages $\gtrsim 10 \mathrm{Gyr}$ ), although non-negligible fractions of younger clusters ( 2-8 Gyr) have been claimed in some cases: NGC 1316, NGC 4365 and NGC 5846. In NGC 1316 the presence of intermediate-age $(\sim 2-3 \mathrm{Gyr})$ clusters (Goudfrooij et al. 2001b) is consistent with its very young luminosityweighted age (Kuntschner 2000). This implies that NGC 1316 has experienced a major merger event $3 \mathrm{Gyr}$ ago (Goudfrooij et al. 2001a). However, in NGC 4365 and NGC 5846 there is no evidence from integrated light (Yamada et al. 2006; and Thomas et al. 2005, respectively) that these galaxies have undergone recent star formation activity.

A general characteristic of GC systems of massive galaxies is having their (optical) colours distributed in a bi(multi)modal way. Spectroscopic studies have suggested that colour 
bimodality is fundamentally due to metallicity differences (e.g. Strader et al. 2007) as most GCs are found to be old (Strader et al. 2005; Cohen et al. 1998), although age differences of $\sim 2$ Gyr are still allowed within uncertainties. The colour distributions of GCs in NGC 4365 and NGC 5846 do not show the bimodality feature (Brodie \& Strader 2006) as clearly as other high mass galaxies. Instead, NGC 4365 has been reported to have a colour distribution with a normal blue peak but a broader red peak shifted towards bluer $(V-I)$ colours (Larsen et al. 2001, 2005 ) if compared to other galaxies. Peng et al. (2006) report bimodality for the $(g-z)$ colour distribution of this galaxy with a broad red peak. The colour distribution of NGC 5846 is bimodal although the distribution of the central clusters shows a red peak with a hint of some additional intermediate colour GCs (Forbes et al. 1997a). Adding the high fraction of intermediate-age GCs to this argument, suggests that the GC systems of these galaxies are not like other similar type ellipticals.

The apparently inconsistent results of NGC 4365 and NGC 5846, with no sign of intermediate-age stellar populations hosting a large fraction of intermediate-age GCs, is intriguing. GC and integrated light studies of the stellar population content of a galaxy should yield compatible conclusions. With this in mind we have studied the ages of GC systems in $14 \mathrm{E} / \mathrm{S} 0$ galaxies through optical/NIR photometry. We compare differentially a large number of GC systems with homogeneous data. The GC systems are also compared with SSP models.

\section{Observations and data}

The homogeneous survey of extragalactic GCs that we carried out with optical and NIR photometry was presented in ChiesSantos et al. (2011). In the same article the data reduction techniques were described in detail, and we will only briefly mention them here. We obtained $K\left(K_{\mathrm{S}}, 2.2 \mu \mathrm{m}\right)$ band imaging with the LIRIS spectrograph and imager (Acosta Pulido et al. 2003; Manchado et al. 2004) at the William Herschel Telescope (WHT) from 2007 to 2009. The $K$ imaging was combined with archival $F 475 W(\sim$ Sloan $g$ ) and F850LP ( Sloan $z$ ) images from the Advanced Camera for Surveys (ACS) on board the Hubble Space Telescope (HST). Our sample is a sub-sample of the SAURON sample (de Zeeuw et al. 2002) and comprises bright elliptical and lenticular galaxies $\left(M_{\mathrm{B}}<19\right)$ with $(m-M)<$ 32. Each galaxy was imaged in the $K$-band for about $3.4 \mathrm{~h}$ and reduced with LIRISDR and standard IRAF routines. The $g$ and $z$ images were reduced with the MULTIDRIZZLE task in the STSDAS package.

The GCs were detected in the ACS images and transformed to the LIRIS coordinate system. Following this, aperture photometry and effective radii $\left(R_{\text {eff }}\right)$ were measured for the cluster candidates, with PHOT (Stetson 1987) and ISHAPE (Larsen 1999), respectively. A final sample of GCs was obtained applying the criteria: $g<23,0.5<(g-z)<2.0,1<R_{\text {eff }}(\mathrm{pc})<$ 15 . Finally, a careful visual inspection was done, where obvious background galaxies still left in the sample were excluded. Sources too close to each other in the ACS images were flagged and if they appeared as one bigger source in the LIRIS frame they were also excluded. We stress that any results for NGC 4382 and NGC 4473 should be taken with caution as their $K$-band observations were obtained in highly extincted conditions.

\section{Deriving ages of GCs}

In this section we attempt to derive ages of GC systems employing two different methods. In Sect. 3.1 we directly compare the
GC systems with SSP models. In Sect. 3.2 we look for age differences without making use of SSP models. The second method gives more promising results.

\subsection{Simple stellar population models}

\subsubsection{Data-model comparison}

In Fig. 1 we plot 2-colour diagrams, $(g-z)$ vs. $(g-K)$ for the final sample of GCs, following the criteria outlined in Sect. 2. Padova SSP models are shown in this plot, with model sequences of constant age ranging from the lower right to the upper left of each panel $(2,3,6,14 \mathrm{Gyr})$ - (see legend in the bottom panels). Lines of constant metallicity are also plotted. Metal-poor clusters are expected to be located in the bottom left part of the diagram whereas metal rich ones should be located in the upper portion. Padova SSPs are retrieved from the CMD 2.2 input form (http: //stev. oapd. inaf.it/cmd), with Marigo et al. (2008) isochrones and are from now on referred to as Padova08 SSPs. Compared with these models, the data appear to be consistent with the oldest track, $14 \mathrm{Gyr}$. Nevertheless, the $6 \mathrm{Gyr}$ intermediate-age track also seems to be a good match. If we exclude NGC 4382 due to highly extincted data, then qualitatively, NGC 4406 seems to host GCs with perhaps younger ages than the rest of the sample, while NGC 4365 does not appear to differ significantly from the other galaxies in terms of the ages of its GCs. Differences and similarities of the GC systems are quantified in Sects. 3.1.2 and 3.2.

In Fig. 2 Maraston (2005) and Charlot \& Bruzual (in prep.) models are overplotted in the 2-colour diagrams, as in Fig. 1. The comparison of the data with these models (different panels of Fig. 2) suggests younger ages than the comparison with the Padova08 models. Therefore, concluding how old or young a GC is through direct comparison to SSP models does depend on the choice of the model. Nevertheless, age estimates determined through Padova08 models are closer to spectroscopically derived ages. This is probably due to the new AGB and TPAGB treatment of Marigo et al. (2008). Maraston (2005) uses a different prescription of the AGB and TP-AGB phases through the fuel consumption theorem. Charlot and Bruzual models use older versions of the Padova tracks (e.g. Padova 1999) which include older calibrations of the AGB phase and do not include a detailed prescription of the TP-AGB phase.

\subsubsection{Blue and red peak ages}

In Fig. 1 the median values of the blue and red populations are shown illustrated with larger symbols and blue and red colours respectively. We have divided each GC system in a red (metalrich) and a blue (metal-poor) sub-population. We have chosen to use the median values as the observational scatter is colour dependant (with blue clusters having more spread) and the median is more robust against outliers. The value which indicates the division between blue and red clusters was determined by means of the KMM test (Ashman et al. 1994). We fit two Gaussians to the $(g-z)$ distributions of the galaxies. The value where the blue and red Gaussians intersect is the $(g-z)$ division value for the sub-populations. We use the mean of this value for the two most cluster-rich galaxies, NGC 4486 and NGC 4649, $(g-z)=1.187$. For NGC 4365 and NGC 4278 the $(g-z)$ distribution was found to be most likely unimodal. For this reason we also plot the median of the whole population with a different colour. The medians of the blue sub-populations have a trend of falling on relative younger tracks than the median of the red 
A. L. Chies-Santos et al.: Age distributions of GCs in early-type galaxies. II.

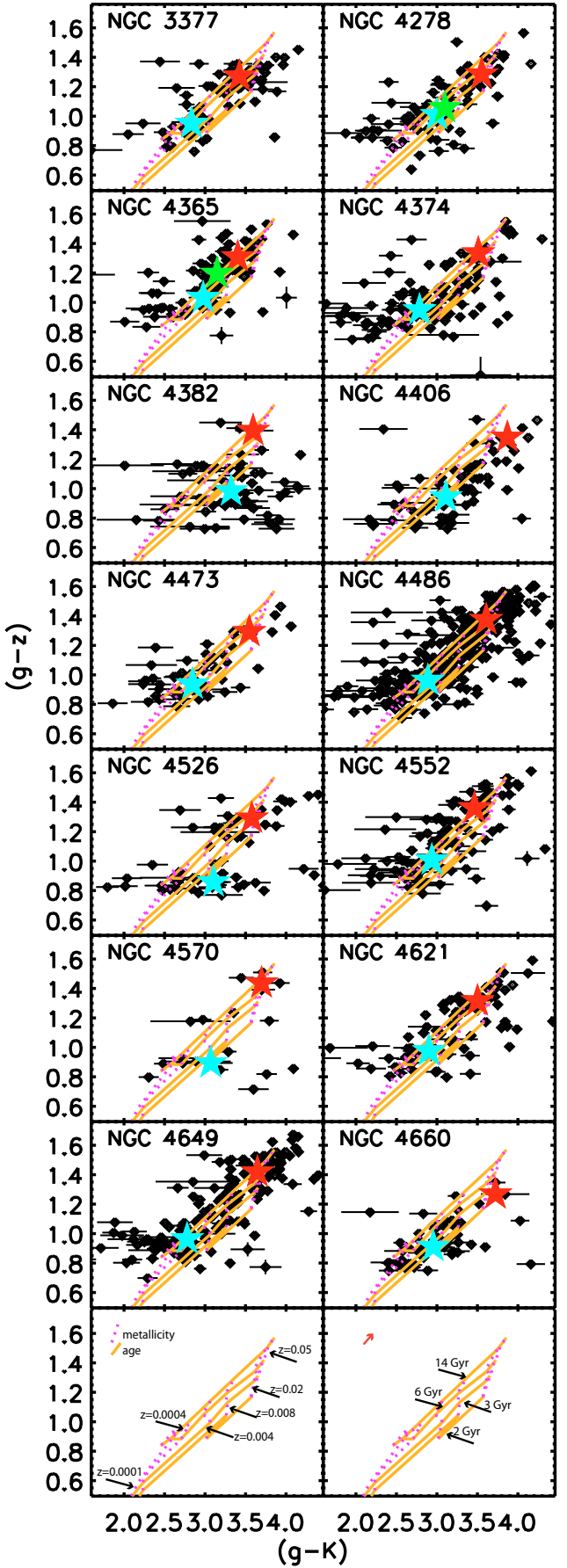

Fig. 1. 2-colour diagrams for the GC systems of the 14 galaxies. $(g-z)$ vs. $(g-K)$. Padova08 SSP models are overplotted with model sequences of constant age and metallicity according to the legend in the bottom panels. The ages increase from the lower right to the upper left. The metallicity tracks increase from the lower left to the upper right. The large blue and red star symbols indicate the medians of the blue and red sub-populations, respectively. They are divided assuming a common division value of $(g-z)=1.187$. The green symbol in the panels corresponding to NGC 4365 and NGC 4278 indicates the median of the single populations as the $(g-z)$ distribution of these galaxies was considered to be more likely unimodal. The red arrow on the bottom most right panel indicates a typical reddening vector.

sub-populations. For example the value which corresponds to the median of the red sub-population of NGC 4486 is located
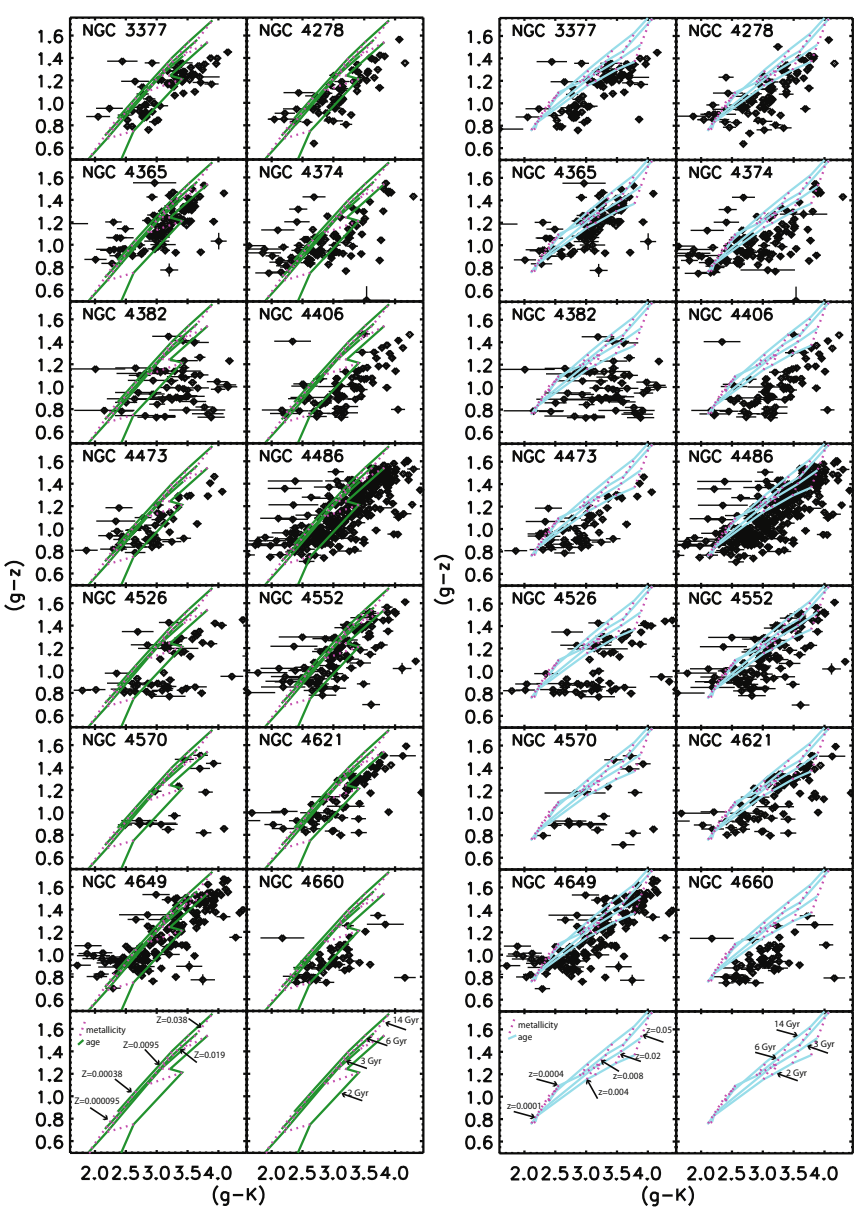

Fig. 2. 2-colour diagrams for the GC systems of the 14 galaxies. $(g-K)$ vs. $(g-z)$, as in Fig. 1: Maraston (2005) with red horizontal branch at the two lowest metallicities (left panel) and Charlot \& Bruzual (in prep.) (right panel).

between the 6 and 14 Gyr SSP while the blue one falls between the 3 and 6 Gyr SSP.

Figure 3 shows a $(g-K)$ vs. $(g-z)$ 2-colour diagram for the GCs of 12 galaxies combined, excluding NGC 4382 and NGC 4473. The blue and red large symbols correspond to the median colour of the blue and red clusters respectively, assuming a fixed dividing colour at $(g-z)=1.187$. This figure shows that the blue population is subject to larger scatter in the $(g-K)$ direction than the red population. This is because blue clusters are generally fainter in $K$, especially due to the selection in the $g$ band. This is also seen in Fig. 1. The finding of on average younger blue GCs as compared to red ones in Figs. 1 and 3 comes from the direct comparison between data and SSP models. We stress that this should be interpreted with caution and verified with spectroscopy and/or with future SSPs. One might think that objects with large $(g-k)$ colours and small error bars (see Fig. 1) would be making the blue population artificially young. Some of these objects could be background stars or foreground galaxies (see e.g. Puzia et al. 2004). We note that a careful visual inspection was performed removing obvious background galaxies and blends (see Chies-Santos et al. 2011). There could still be some contaminants left, but only spectroscopic analysis of such objects would give clearer answers.

In order to quantify the ages that best match the GC systems we ran AnalySED (Anders et al. 2004) on the median magnitudes of the sub-populations, presented in Fig. 1. This code 


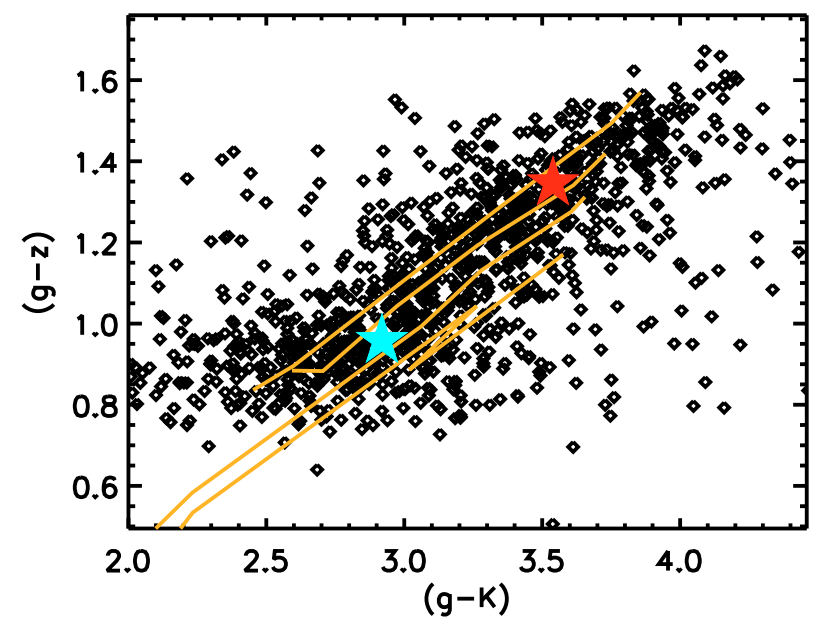

Fig. 3. The $(g-K)$ vs. $(g-z)$ 2-colour diagram for the GCs of 12 galaxies combined, excluding NGC 4382 and NGC 4473. The blue and red large symbols correspond to the median colour of the blue and red clusters respectively. The orange lines represent Padova08 SSPs of 2, 3,6 and 14 Gyr, like in Fig. 1.

finds the best age and metallicity fits using model spectral energy distributions (SEDs). We used as input SEDs Padova08 SSPs integrated colours and kept the extinction fixed. For simplicity, Padova08 models starting at $1 \mathrm{Gyr}$ were employed. At old ages, metallicity has a much stronger effect on the SEDs than does age, as seen in the 2-colour diagrams shown before. Therefore, to get meaningful results it is important to have a grid in metallicity with sufficient resolution. For Padova08 SSPs one can download a grid of metallicities with a spacing that suits the user.

In Fig. 4 the best age and metallicity fits returned by AnalySED are shown. The error bars represent the standard error on the mean. We have used a metallicity grid with a spacing of $\Delta Z=0.0015$. The ages derived by AnalySED vary between 1 and $14 \mathrm{Gyr}$, i.e., the data is roughly consistent with the whole range of models. The upper limits in most cases, however, appear to match our expectations (from spectroscopy, e.g. Strader et al. 2005; Cenarro et al. 2007) of the age of GCs in early type galaxies consistent with $\sim 10 \mathrm{Gyr}$. We strongly caution against interpreting the ages derived in the present section as real ages, they only serve to give an impression of what one gets from a literal comparison of the data with SSP models. Mean ages are well below $10 \mathrm{Gyr}$, formally the mean blue is $\sim 3 \mathrm{Gyr}$ while the mean red is $\sim 8$ Gyr. Note that this is the same qualitative conclusion reached with Figs. 1 and 3: the blue population is on average younger than the red one when using the Padova08 SSPs as a reference. The corresponding best metallicity fits are shown in the right panel of Fig. 4. The median blue best fit is $Z=0.00046([\mathrm{Fe} / \mathrm{H}] \sim-1.61 \mathrm{dex})$ and the red is $Z=0.00121([\mathrm{Fe} / \mathrm{H}] \sim-1.19 \mathrm{dex})^{1}$. The metal-poor peak value is comparable, or somewhat lower than what is found for typical GC systems of massive galaxies. For example Cohen et al. (1998) finds the blue and red metallicity peak values for the NGC 4486 GC system to be -1.3 and -0.7 dex respectively, with a dispersion of 0.3 dex. More recent measurements (Brodie $\&$ Strader 2006) agree on the blue peak value, -1.3 but the red peak is significantly more metal-rich, $-0.2 \mathrm{dex}$.

$\overline{1 \text { The approximation }[\mathrm{Fe} / \mathrm{H}]} \sim \log \left(Z / Z_{\odot}\right)$ and $Z_{\odot}=0.019$ were used.

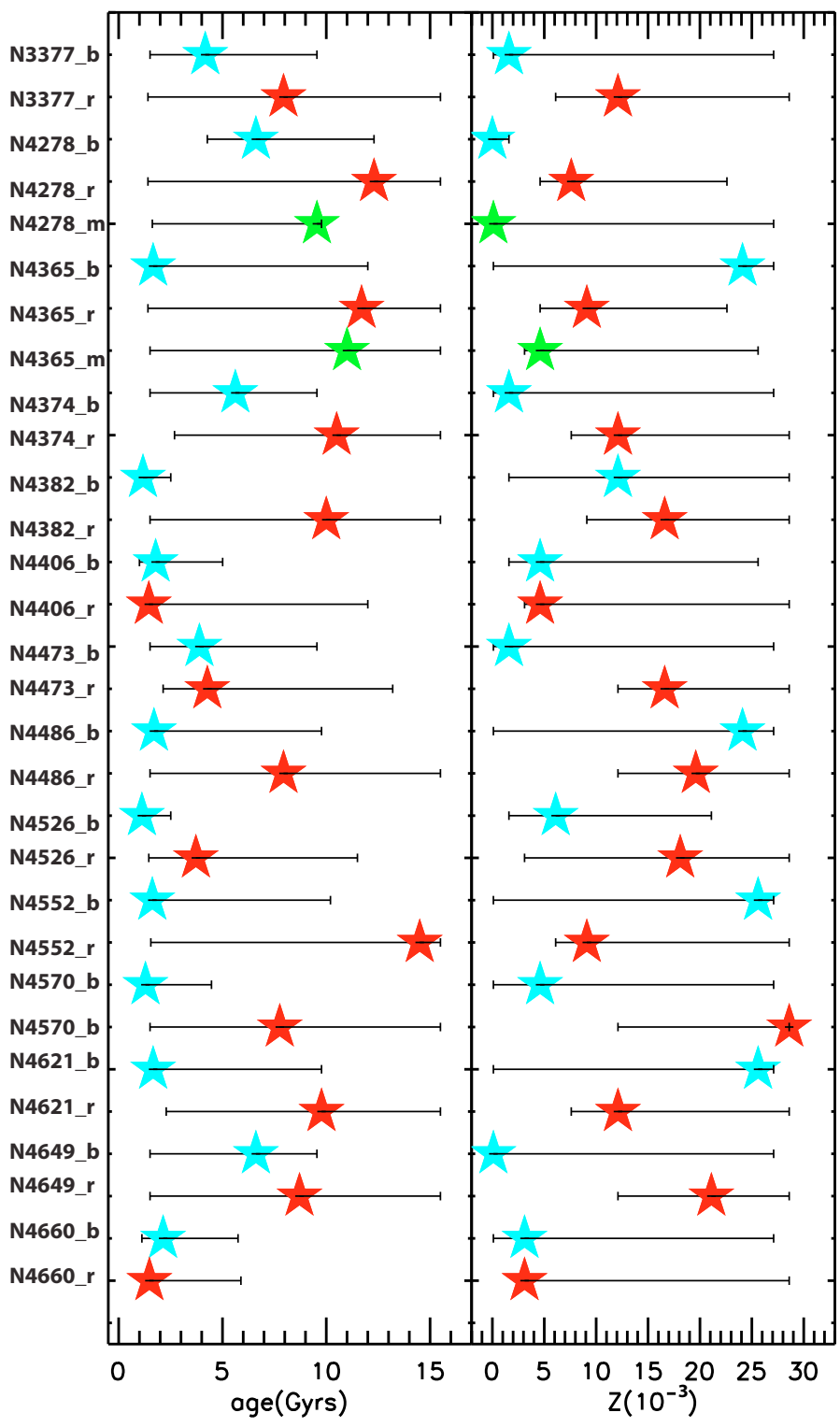

Fig. 4. The best age and metallicity fits returned by AnalySED with Padova08 SSPs for the median of the blue (metal-poor) and red (metalrich) sub-populations indicated by blue and red symbols respectively. The median of a single population for NGC 4365 and NGC 4278, where KMM rejected the bimodal fit as opposed to the unimodal one, is indicated by a green star.

\subsection{Relative comparison without SSP models}

Until up to this point we have attempted to derive ages by direct data model comparison. It is well documented in the literature that a direct comparison of integrated GC colours with SSP models tends to yield ages that are significantly younger than a Hubble time (e.g. Larsen et al. 2005). While this effect is less pronounced in the most recent Padova SSP models compared to earlier models, we still find it difficult to believe the very young ages assigned to the blue GCs in particular (Fig. 4). It is worth noting that the spacing between isochrones of different ages remains small even when including near-infrared colours (Fig. 1), so even small errors in the model colours will have a large effect on the ages. We expect that as the models continue to be refined, these offsets will grow smaller. In the meantime, it may be fruitful to explore other ways of determining age differences between GC systems in different galaxies. We therefore 
A. L. Chies-Santos et al.: Age distributions of GCs in early-type galaxies. II.

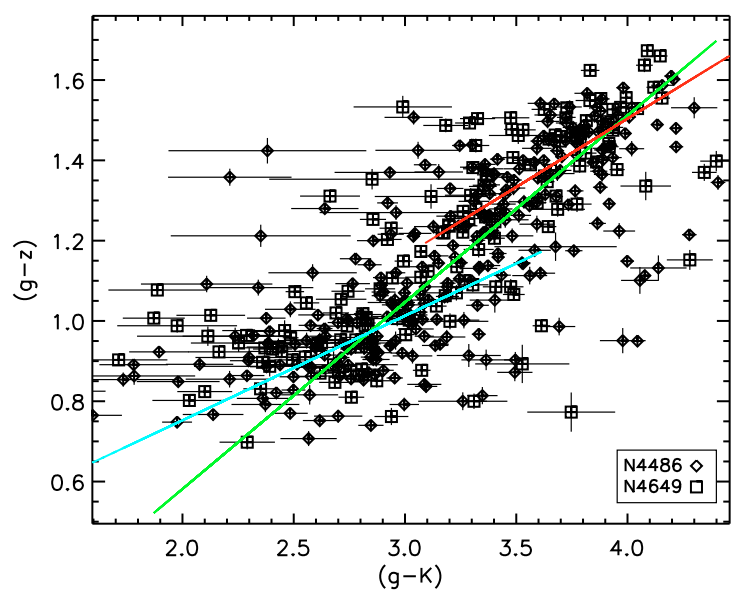

Fig. 5. $(g-K)$ vs. $(g-z)$ for the joint GC systems of NGC 4486 and NGC 4649. The weighted best fit for the whole sample, given by Eq. (1) is over plotted in green. The best fit lines for the blue and red subpopulations, given by (2) and (3) are shown in blue and red respectively.

now turn to a purely differential comparison, independent of any SSP models.

Through spectroscopy, the median age distribution of the GC system of NGC 4486 is reported to be 13 Gyr with a dispersion of $2 \mathrm{Gyr}$ (Cohen et al. 1998). The GCs of NGC 4649 are distributed in a similar way in the $(g-K)$ vs. $(g-z)$ plane to those of NGC 4486. Since these two GC systems are both thought to be old and have high numbers of GCs (167 and 301 clusters respectively) we take them as the fiducial old systems. We fit linear relations to the data in the $(g-K)$ vs. $(g-z)$ plane. These fits give more weight to the objects with smaller observational errors in both the abscissa and the ordinate.

In Fig. 5 this best-fit relation given by Eq. (1) is shown. In the same plot, the best fit for the blue and red sub-populations are also shown. These are given by Eqs. (2) and (3) respectively. Eq. (2) is valid for $(g-z)<1.187$ and Eq. (3) for $(g-z)>$ 1.187. One possible reason for the apparent discontinuity of the $(g-z)$ vs. $(g-K)$ relations of the blue and red GCs is that the horizontal branch morphology is expected to change abruptly at intermediate colours-metallicities, as discussed in Sect. 4.2. A separation into blue and red best fit lines is taking this into account.

$$
\begin{aligned}
& (g-z)=0.465 *(g-K)-0.349 \\
& (g-z)_{b}=0.260 *(g-K)_{b}+0.232 \\
& (g-z)_{r}=0.340 *(g-K)_{r}+0.140 .
\end{aligned}
$$

In Fig. 6 the best fit lines (1), (2) and (3) in the ( $(g-K)$ vs. $(g-z)$ plane for the joint GC systems of NGC 4486 and NGC 4649 are over plotted for the different galaxies. Also plotted are the median colours for the blue and red sub-populations of the respective galaxies. This plot shows qualitatively how much and in what direction the mean of the sub-populations deviates from the lines in Fig. 5. For instance, the median of the blue and red populations of some GC systems, such as NGC 4406, deviate significantly to the right of the green line.

In the following, we introduce a new method to derive relative ages of GC systems with the purpose of quantifying the magnitude and direction of the deviation of a GC from Eqs. (1)-(3). In Sect. 3.1 we showed that relative to the SSP models, a younger cluster falls on the lower right of the $(g-K)$ vs. $(g-z)$ plane while an older cluster falls on the upper

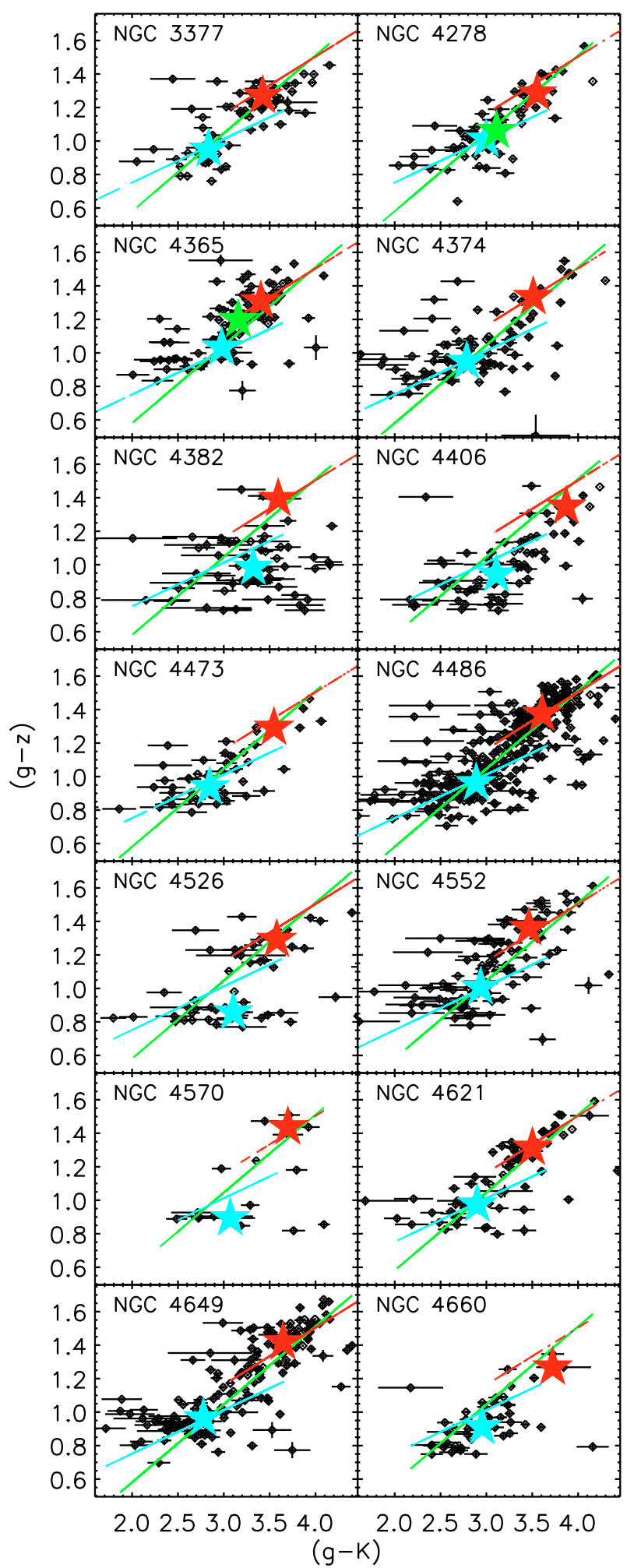

Fig. 6. Same as Fig. 1 but with the Padova08 SSPs replaced with the best fit relations given by Eqs. (1)-(3) shown in Fig. 5 over plotted.

left of this diagram. By considering the green line as an SSP, with metallicities increasing from the lower left to the upper right, one can make statements about relative ages of GCs with respect to this line. We define $\delta, \delta_{\text {blue }}$ and $\delta_{\text {red }}$ as the projected distances of a GC from relations (1)-(3) respectively. These, are given by:

$\delta=\frac{0.465 *(g-K)-(g-z)-0.349}{\sqrt{0.465^{2}+1}}$ 


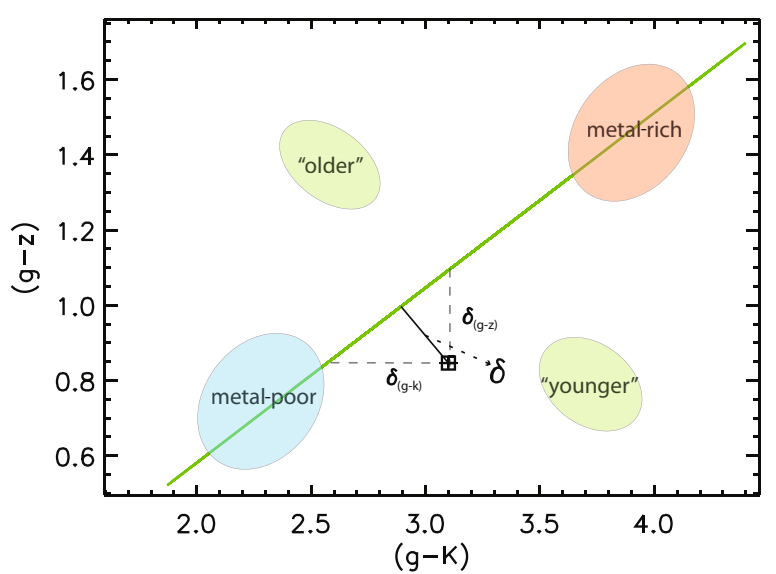

Fig. 7. A cartoon illustrating the definition of $\delta$ in the $(g-K)$ vs. $(g-z)$ diagram for one cluster (see text for details) and the location of the "younger" and "older" objects relative to the green line. The location of metal-poor and metal-rich objects is also shown.

$\delta_{\text {blue }}=\frac{0.260 *(g-K)-(g-z)+0.230}{\sqrt{0.260^{2}+1}}$

$\delta_{\text {red }}=\frac{0.341 *(g-K)-(g-z)+0.140}{\sqrt{0.341^{2}+1}}$.

Figure 7 shows a cartoon illustrating $\delta$ for a cluster in the $(g-K)$ vs. $(g-z)$ plane. In this figure the location of younger and older clusters relative to this line as well as the location of the metalrich and metal-poor clusters are plotted. The values of $\delta$ are age tracers of GCs in this plane. An object falling to the right of the green line will have a positive $\delta$ value. A positive $\delta$ value means that this object has an age that is younger than the age that the green line traces $(\delta=0)$. In this sense, a GC system which lies further to the right of $\delta=0$ (and thus has a larger positive delta value) has the best chance of hosting younger GCs

In Fig. 8 the histograms for the $\delta$ values of the different galaxies are shown. By comparing the different $\delta$ distributions one can check if there are age differences between the different distributions. It is readily seen that not all GC systems are consistent with a $\delta=0$. For example, note that the peak of the NGC $4406 \delta$ distribution is $\sim 0.2$ whereas the peak of the NGC 4649 is $\sim-0.05$. By taking the mean value of the distributions of $\delta$ it is possible to see how on average the different $\mathrm{GC}$ systems are younger (or older) from the joint GC system of NGC 4486 and NGC 4649. In Fig. 9 a diagram shows the mean (and median) of $\delta$ with the standard error on the mean for each GC system. The usual interpretation of any offset from $\delta=0$ would be that there are (mean) age shifts. The larger shifts occur for $\delta>0$, i.e. to younger ages. Therefore the GC system of NGC 4406 is on average younger than the GC system of NGC 4649 and NGC 4486. The best cases for hosting younger GCs are NGC 4382, NGC 4406, NGC 4526, NGC 4570 and NGC 4660. All of them are S0s, except for NGC 4660 which is classified as an E5 (NED). However, this galaxy is also quoted to be an E3/S0 by Ferrarese et al. (2006) who report boxy isophotes and a faint blue structure of about $2.5^{\prime}$ with two spiral arms within an ACS pointing. Recently, Kormendy et al. (2009) argued that NGC 4660 is an S0. This galaxy hosts a disc, although it contributes relatively little to its light.

By performing the $\delta$ analysis for the different GC systems separately for blue and red clusters one can check which subpopulation has a greater $\delta$ shift. The means (and medians) of

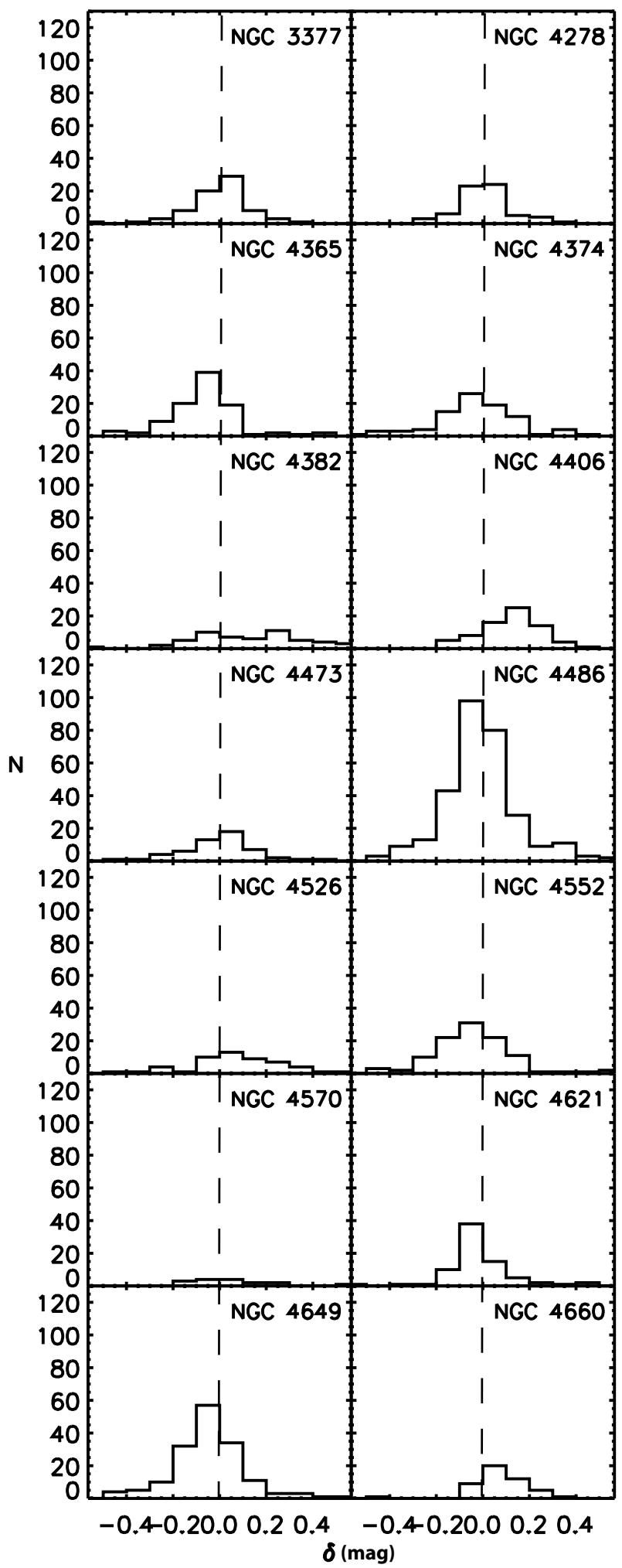

Fig. 8. The $\delta$ distribution: the difference between the GCs and the best fit line for the whole sample given by Eq. (1). The dashed line indicates the value $\delta=0$.

the $\delta_{\text {blue }}$ and $\delta_{\text {red }}$ distributions are plotted in Fig. 10. The values of $\delta_{\text {blue }}$ and $\delta_{\text {red }}$ are based on the colour ranges where Eqs. (2) and (3) are valid. The blue populations of NGC 4570, NGC 4526, NGC 4406 and NGC 4382 have the greatest shifts, $\delta_{\text {blue }} \gtrsim 0.1$. The red populations on the other hand, have a 
A. L. Chies-Santos et al.: Age distributions of GCs in early-type galaxies. II.

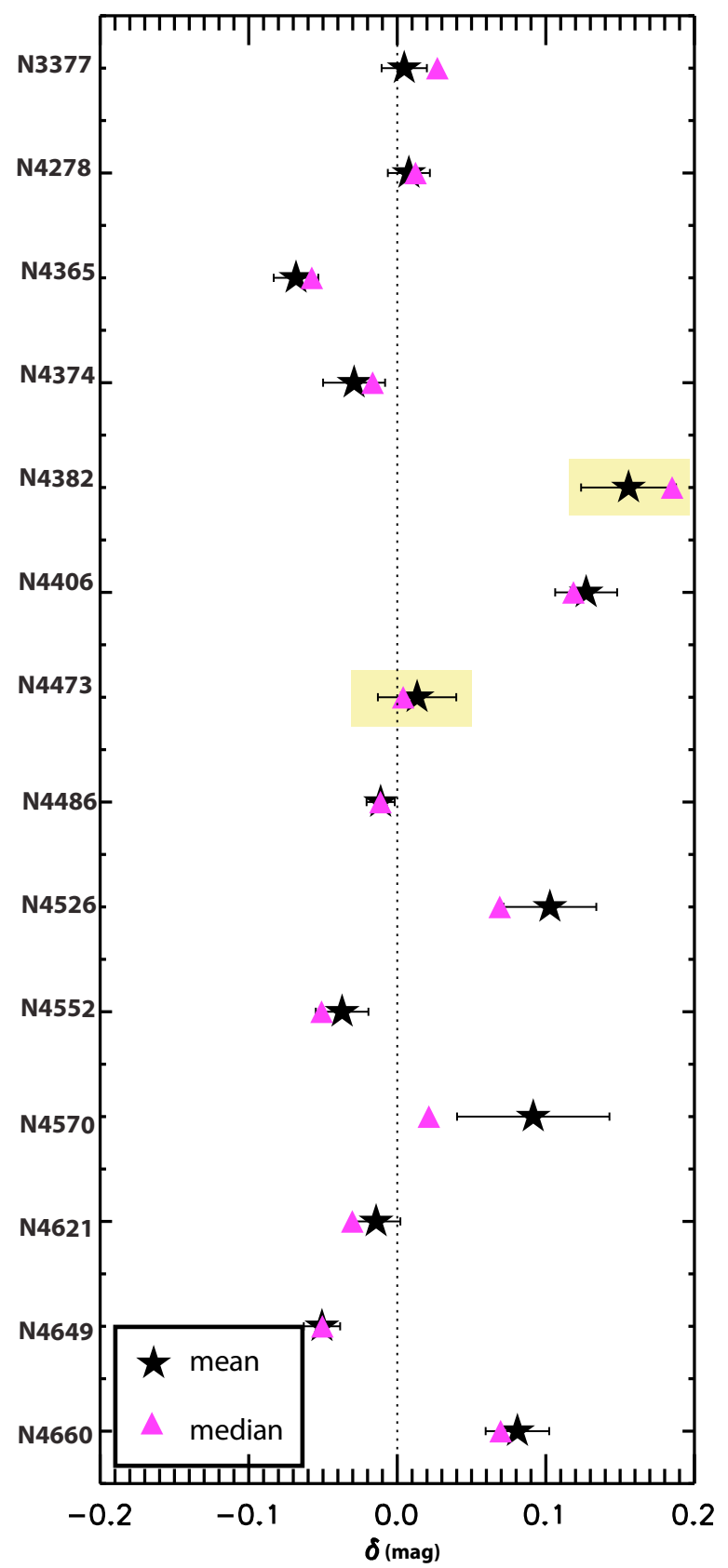

Fig. 9. The mean and median of the $\delta$ distribution for the GCs of different galaxies, shown in Fig. 8. The error bars centred in the mean indicate the standard error on the mean of the quantity $\delta$. The dotted line stands for $\delta=0$. NGC 4382 and NGC 4473 are marked with yellow rectangles because their $K$-band data were obtained in highly extincted conditions.

smaller, apparent shift. Moreover, the $\delta_{\text {blue }}$ offsets from $\delta_{\text {blue }}=0$ in Fig. 10 are very similar to the $\delta$ offsets from $\delta=0$ in Fig. 9.

It is important to check how much scatter around the vertical line of $\delta, \delta_{\text {blue }}$ and $\delta_{\text {red }}=0$ might be attributed to zero point, photometric and aperture correction uncertainties. The accuracy of the LIRIS $K$-band photometric calibration is estimated to be $\sim 0.03-0.05 \mathrm{mag}-$ (see Sect. 3.2 of Chies-Santos et al. 2011). There are cases where the mean $\delta$ reaches values as large as three times the values of the uncertainty on the photometric calibration. Therefore only part of the scatter in Figs. 9 and 10 could be attributed to uncertainties in the $K$-band photometric calibration.

While measurements of the mean $\delta$ parameter in different galaxies reveal some interesting differences, we have not yet

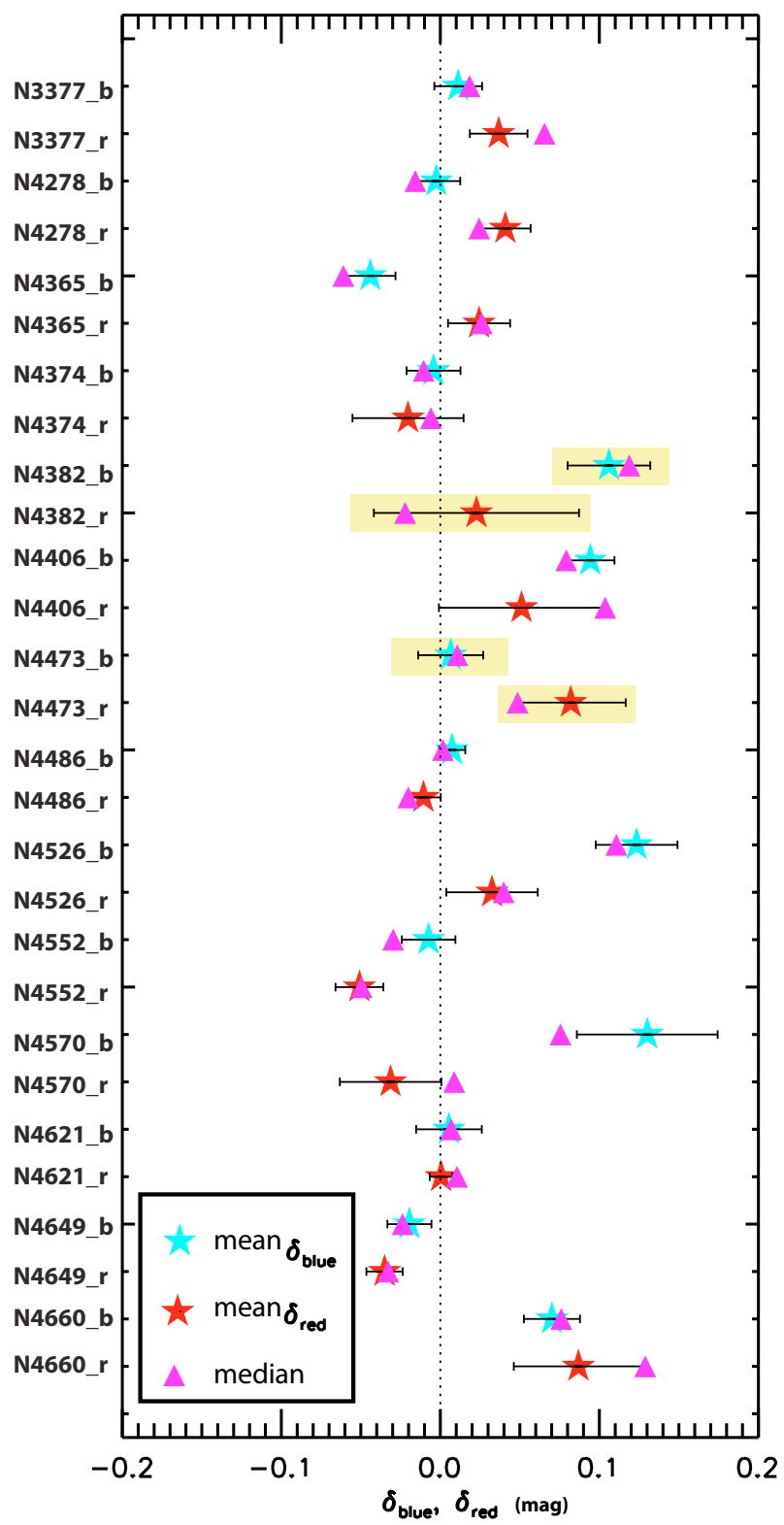

Fig. 10. Similar to Fig. 9 but for the mean and median of the $\delta_{\text {blue }}$ and $\delta_{\text {red }}$ distributions for the GCs of different galaxies. The error bars centred in the mean indicate the standard error on the mean of the quantities. The dotted line stands for the value $\delta_{\text {blue }}$ and $\delta_{\text {red }}=0$. NGC 4382 and NGC 4473 are marked with yellow rectangles because their $K$-band data were obtained in highly extincted conditions.

discussed how to interpret this in terms of actual age differences. To this end, we are guided by a comparison with SSP models. In Fig. $11(g-K)$ vs. $(g-z)$ 2-colour diagrams are shown with lines of constant $\delta$. Padova08 SSP models are also overplotted, as in Fig. 1. Note that the best fit line (1), is roughly consistent with the $6 \mathrm{Gyr}$ track. Moreover the line of $\delta \sim 0.1$ is located between the 2 and 3 Gyr old tracks. This means that an offset of $\delta \sim 0.1$ is roughly consistent with an upper limit of $\Delta$ age $\sim 4$ Gyr. $\delta$ values consistent with $\sim 0.05$ fall between the 3 and 6 Gyr old tracks, giving a maximum $\Delta$ age $\sim 3 \mathrm{Gyr}$. If we instead shift the model grid such that the 14 Gyr SSP model coincides with the $\delta=0$ line, an offset of $\delta=+0.1$ would correspond to an age of about $6 \mathrm{Gyr}$, or a $\Delta$ age of $8 \mathrm{Gyr}$. It is thus clear that a translation of our $\delta$ parameter to an actual age difference is quite uncertain. We stress that care must be taken with the interpretation of the 


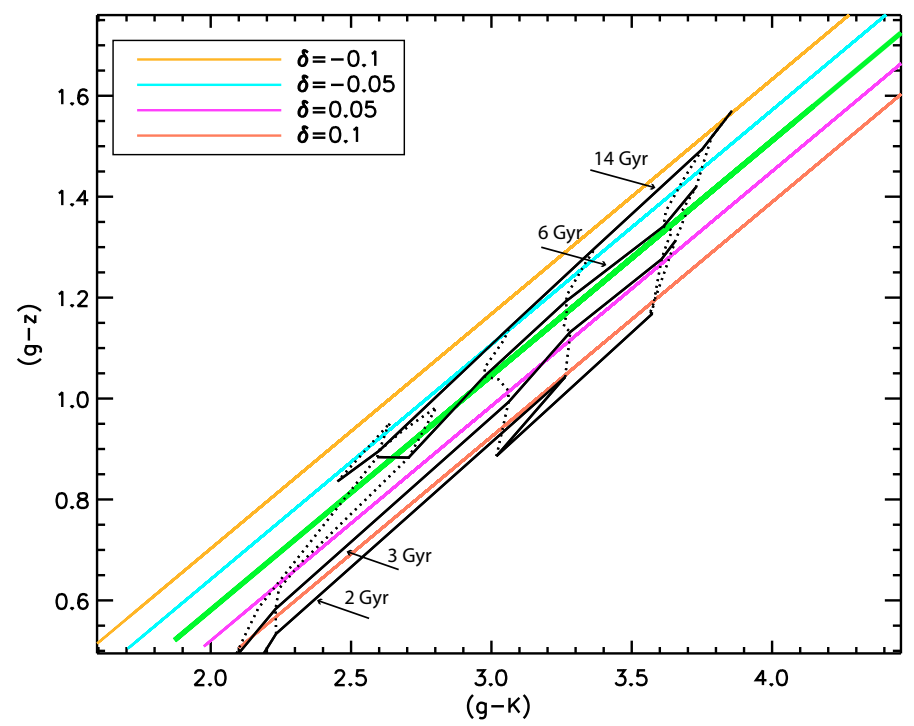

Fig. 11. $(g-K)$ vs. $(g-z)$ diagrams showing lines of constant $\delta$, according to the legend. Padova08 SSP models are over plotted as in Fig 1. The green line is the best-fit line for the joint GC systems of NGC 4486 and NGC 4649, given by relation (1).

$\delta$-parameter as a pure proxy for age, due to the age-metallicity degeneracy. For instance, the SSP tracks in the 2-colour diagram do not run parallel to the lines of constant $\delta_{\text {blue }}$ and $\delta_{\text {red }}$ and the comparison between the two is metallicity dependant. Therefore it is difficult to even roughly estimate what $\delta_{\text {blue }}$ and $\delta_{\text {red }}$ mean in terms of $\Delta$ age.

One might ask whether differences in the $\delta$ values could be related to metallicity rather than age effects due to the known peak GC metallicity vs. galaxy luminosity relation (e.g. Brodie $\&$ Strader 2006). This would happen because the fiducial best-fit lines Eqs. (1)-(3) are defined on two of the highest luminosity galaxies in our sample. We argue this does not appear to be the case. The reason is that the peak GC metallicity vs. galaxy luminosity relation does not translate into a peak GC metallicity morphology relation. For example, the S0 NGC 4406 which has a higher delta value has a $M_{\mathrm{B}}$ value comparable to that of galaxies with low delta values, e.g. NGC 4365. Another example is NGC 4382 which has a high delta value and an $M_{\mathrm{B}}$ value consistent with that of NGC 4649. Although the case for NGC 4382 is to be taken with caution due to the lower-quality $K$-band data. Moreover, we have calculated the $\delta_{\text {blue }}$ for two clusters with the same age $(10 \mathrm{Gyr})$ and with host galaxy luminosities, $M_{\mathrm{B}}=-21(Z \sim 0.0008)$ and $M_{\mathrm{B}}=-19 \mathrm{GC}(Z \sim 0.0006)$, following Fig. 13 of Peng et al. (2006). This was done in order to test for the worst case scenario, the influence of the peak GC metallicity vs. galaxy luminosity relation on the measured $\delta$ values. By making use of Padova08 SSPs we find that the difference between these $\delta_{\text {blue }}$ values is 0.0064 , i.e., much smaller than the actual delta values. Therefore, the peak GC metallicity vs. galaxy luminosity relation has no significant impact on the $\delta$ values over the luminosity range relevant for our sample.

\subsection{GC ages correlated with host galaxy morphological type}

In Fig. 12 the mean (and median) of $\delta, \delta_{\text {blue }}$ and $\delta_{\text {red }}$ are plotted against the morphological type of the galaxy. The morphological classification of the galaxies is given in Table 1 . We do not adopt the Kormendy et al. (2009) classification because it is not available for all galaxies. Besides NGC 4660, three other

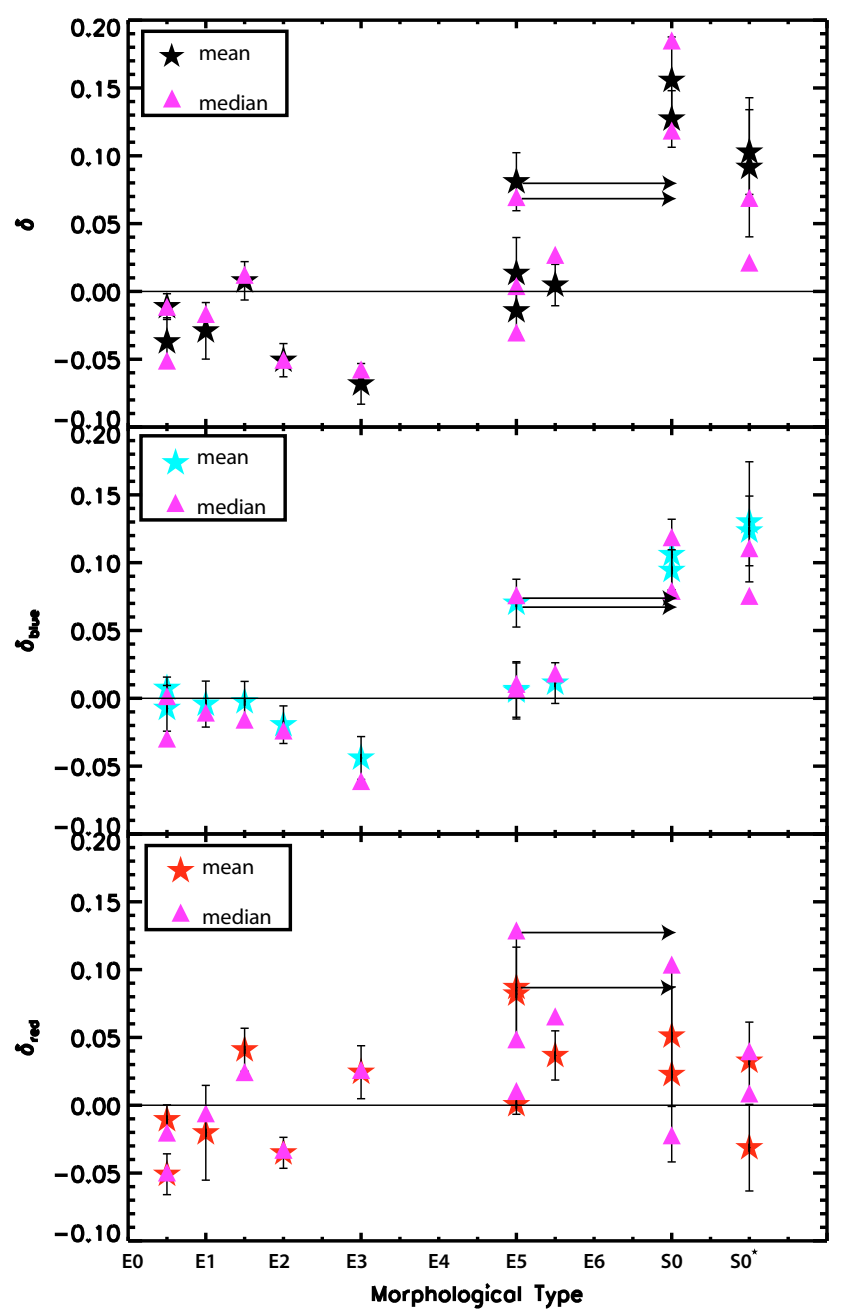

Fig. 12. The mean and median of $\delta, \delta_{\text {blue }}$ and $\delta_{\text {red }}$ as a function of morphological type in the different panels. The solid line indicates the value where the mean of the GCs would not differ from the relations (1)-(3). The arrows indicate the location of the values for NGC 4660 when classified as S0 instead of E5.

cases are classified differently in this study: NGC 4406 is classified as an E3, NGC 4382 as an E2 and NGC 4473 as an E4. We adopt the classification from the SAURON studies which include all the galaxies in our sample (e.g. Emsellem et al. 2007). It is readily seen that the mean of $\delta$ correlates with galaxy morphology. The $\delta$ (and $\delta_{\text {blue }}, \delta_{\text {red }}$ ) distributions of the galaxies of later Hubble type (E5, S0's, S0*'s) have on average larger positive offsets from $\delta=0$ (and $\delta_{\text {blue }}=0, \delta_{\text {red }}=0$ ) than galaxies of earlier Hubble type (E0's). These offsets are interpreted as being due to younger (on average) GC ages. If NGC 4660 were to be classified as an S0, this correlation would be even stronger. To quantify the strength of this correlation we make use of the Spearman's rank correlation coefficient $(\rho)$ which is a nonparametric measure of statistical dependence between two variables. If one of the variables is a perfect monotone of the other, then the quantity $\rho$ will be equal to +1 for correlation and -1 for anticorrelation. Considering NGC 4660 as an E5(S0) in the relation of Fig. 12, the values of $\rho$ are: $0.75(0.76)$ for the mean and $0.69(0.73)$ for the median. For $\delta_{\text {blue }}, \rho=0.79(0.81), 0.81(0.83)$ and for $\delta_{\text {red }}, \rho=0.35(0.41), 0.47(0.53)$ for the mean and median respectively. If one removes NGC 4382 and NGC 4473, due to highly extincted $K$-band photometry, the $\rho$ values become 0.67 , 
A. L. Chies-Santos et al.: Age distributions of GCs in early-type galaxies. II.

Table 1. Galaxy (1), morphological type from Emsellem et al. (2007), Cappellari et al. (2007) and NED and (2) morphological type adopted for Figs. 12 and 13.

\begin{tabular}{ccc}
\hline \hline Galaxy & $\begin{array}{c}\text { Morphological } \\
\text { type } \\
(2)\end{array}$ & $\begin{array}{c}\text { Morphological } \\
\text { type (plots) } \\
(3)\end{array}$ \\
\hline NGC 3377 & E5/E6 & E5/E6 \\
NGC 4278 & E1/E2 & E1/E2 \\
NGC 4365 & E3 & E3 \\
NGC 4374 & E1 & E1 \\
NGC 4382 & $\mathrm{S}^{+}(s) p e c$ & S0 $^{\star}$ \\
NGC 4406 & S0 & S0 \\
NGC 4473 & E5 & E5 \\
NGC 4486 & E0/E1 ${ }^{+} p e c$ & E0/E1 \\
NGC 4526 & SAB0 ${ }^{0}(s)$ & S0 \\
NGC 4552 & E0/E1 & E0/E1 \\
NGC 4570 & S0 sp & S0 \\
NGC 4621 & E5 & E5 \\
NGC 4649 & E2 & E2 \\
NGC 4660 & E & E5 \\
\hline
\end{tabular}

0.63. For $\delta_{\text {blue }}$ the $\rho$ values are $0.74,0.76$ and for $\delta_{\text {red }}$ they are 0.41 and 0.65 , for the mean and median respectively. The values of $\rho$ for $\delta$ (and $\delta_{\text {blue }}$ ) are close enough to 1 for the correlations between $\delta$ (and $\delta_{\text {blue }}$ ) and galaxy morphology to be significant.

From Fig. 11 we have that an offset of $\delta=0.1$ is roughly consistent with a $\Delta$ age of $\sim 4-8$ Gyr. If one considers the median age of $\sim 13 \mathrm{Gyr}$ found by Cohen et al. (1998) for the GC system of NGC 4486, an E0, the average ages of S0 GC systems are extrapolated to be $\sim 5-9$ Gyr. We stress that this is a very rough estimate, given the zero-point problem of the models.

\section{Investigating effects other than age and metallicity in the 2-colour diagrams}

In the top panel of Fig. 13 the standard deviation $(\sigma)$ of the clusters around relation (1) along $(g-K)$ in the $(g-K)$ vs. $(g-z)$ plane is shown as a function of morphological type. Because the photometric errors are dominated by the $K$-band photometry, the scatter in $(g-K)$ will be very nearly proportional to the scatter in $\delta$ if photometric errors are the only cause of offsets from the line defining the $\delta$ parameter. It is immediately seen that the scatter varies among different systems and that in most cases it is larger than the $1 \sigma$ of $\sim \pm 0.4$ permitted (Chies-Santos et al. 2011) from observational uncertainties. Analogously the scatter of $\delta_{\text {blue }}$ and $\delta_{\text {red }}$ along $(g-K)$ as function of galaxy morphology are shown in the middle and in the bottom panels respectively. It is readily seen that the scatter is greater for $\delta$ than for $\delta_{\text {blue }}$ and $\delta_{\text {red }}$ separately. For $\delta$, the mean $\sigma$ is $0.643 \pm 0.007$. The error quoted is the standard error on the mean. For $\delta_{\text {blue }}$ the mean is $0.518 \pm 0.013$ and for $\delta_{\text {red }} 0.403 \pm 0.015$. The scatter becomes smaller when looking at $\delta_{\text {blue }}$ and $\delta_{\text {red }}$ separately. In fact, it seems almost consistent with the observational errors. This suggests that a single linear fit in the 2-colour $(g-K)-(g-z)$ space is an over approximation. By making the division in two linear regimes, it seems that almost all the scatter can be explained by observational errors.

One might think that the relation shown in Fig. 12 might be an artefact of our sky subtraction method through ellipse model fits (see Chies-Santos et al. 2011). Even though the direction the morphology becomes more complicated is the same direction the ellipse model fits became more difficult, Fig. 13 shows that there is no relation between the scatter in $\delta\left(\delta_{\text {blue }}\right.$ and $\left.\delta_{\text {red }}\right)$ and

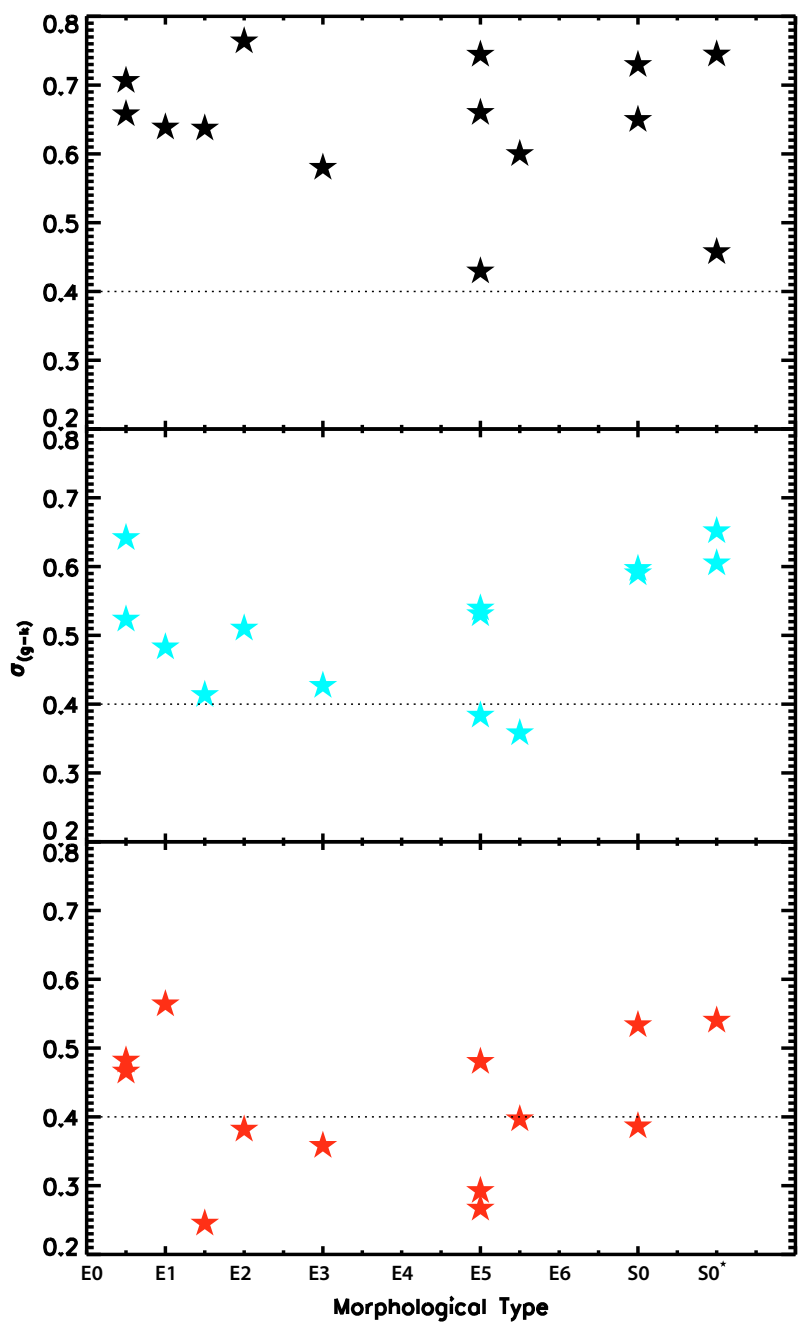

Fig. 13. The scatter represented by the standard deviation $(\sigma)$ for $\delta$, $\delta_{\text {blue }}$ and $\delta_{\text {red }}$ in the $(g-K)$ direction as a function of morphological type. The dotted lines indicate the upper limit to the maximum scatter from observational errors.

the host galaxy morphological type. We therefore conclude that the observed trend of increasing $\delta$ with Hubble type is real.

In Fig. 14 a diagram with the mean scatter for all the GC sample, and for GCs brighter than $K=20$ and $K=19$ is shown. The scatter is now also represented by the median absolute deviation ( $\mathrm{mad}$, indicated by squares) in addition to the $\sigma$ (represented by stars). Both mean mad and $\sigma$ decrease when brighter magnitude intervals are considered. This is true when considering the total combined population as well as the separate red and blue sub-populations. For the combined population the values of $\sigma$ remain greater than the observational errors of $\sim 0.4$ (see Sect. 4 in Chies-Santos et al. 2011), even for a sample with $K<19 \mathrm{mag}$. For the blue and red sub-populations amongst the brighter magnitude samples, this is not the case any longer. The $\sigma$ is consistent with $\sim 0.4$ for the blue and drops below $\sim 0.4$ for the red sub-population. However, a sample binned to brighter magnitudes will of course have a smaller mean observational error value. For $K<19 \mathrm{mag}$ this value is $\sim 0.2$. The $\sigma$ values for $K<19$ mag are much larger than this value for the combined and for the red and blue sub-populations. Even though the mad values are closer to $\sim 0.2$ for the blue and red sub-populations, the observational errors are really $\sigma$ values and not mad values. Therefore there has to be something else responsible for this extra scatter. 


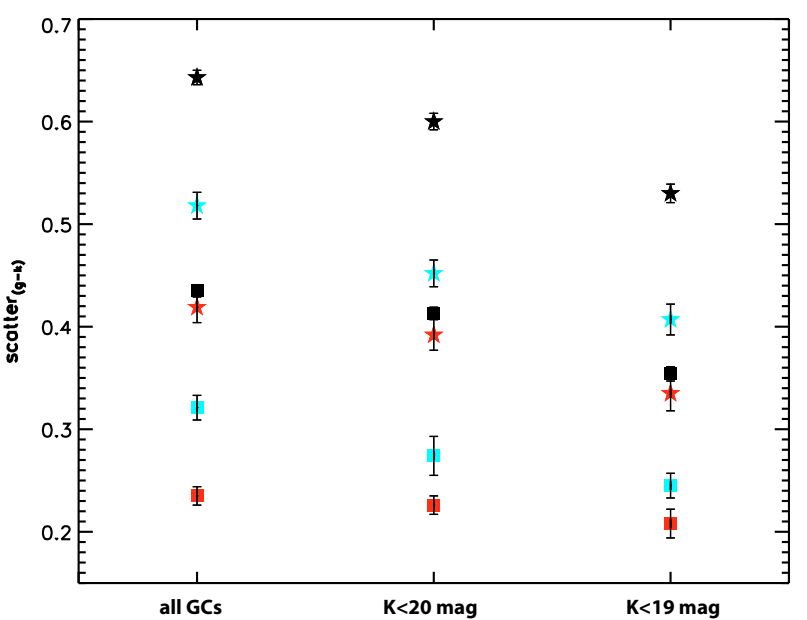

Fig. 14. The mean $\sigma$ (stars) and the mean mad (squares) for the measurements in Fig. 13 for $\delta$ (black symbols), $\delta_{\text {blue }}$ (blue symbols) and $\delta_{\text {red }}$ (red symbols). The error bars are the standard error on the mean.

In this section we investigate which effects other than age and metallicity could be responsible for this extra scatter, and whether these are expected to contribute significantly to any scatter.

\section{1. $\alpha$-enhanced vs. solar-scaled tracks}

What is the effect of $\alpha$-enhancement on the integrated colours of SSPs? For NGC 4472 and NGC 4486 GCs (Cohen et al. 2003 and Cohen et al. 1998) $[\alpha / \mathrm{Fe}]$ estimates were derived to be $\sim+0.3$. Puzia et al. (2005) finds an $[\alpha / \mathrm{Fe}] \sim+0.4$ for their sample of extragalactic GCs. In the recent study of Sharina et al. (2010) it is found that the $[\alpha / \mathrm{Fe}]$ ratios of GCs tend to be higher on average for giants than for dwarf galaxies. If a gE has recently accreted nearby dwarfs with its GCs, they should have therefore lower $[\alpha / \mathrm{Fe}]$ ratios than the GCs formed within the protogalaxy. Salaris \& Cassisi (2007) discuss $\alpha$-enhancement issues in the study of extragalactic GCs through broad-band photometry (see their Fig. 12) and conclude that the net effect of using an $\alpha$-enhanced calibration in GCs with a solar metal mixture is to assign too young ages, especially at the reddest end. These age differences are of the order of $\sim 2$ Gyr. If, on the other hand, one uses a solar scaled calibration to derive ages of $\alpha$-enhanced GCs, the ages assigned will be too old.

Recently the BaSTI group made available $\alpha$-enhanced $([\alpha / \mathrm{Fe}] \sim+0.35)$ isochrones and spectra. These were downloaded from their website and used as input in GALEV to produce $\alpha$-enhanced calibrated SSPs for the $g, z$ and $K$ colours. In Fig. 15 we plot 2 and 10 Gyr SSPs in a 2-colour diagram with solar-scaled and $\alpha$-enhanced metal mixtures. The different tracks are so close together that it is very hard to tell them apart. The magnitude differences seen are too small to account for any noteworthy difference between the two sets of models. The fact that Salaris \& Cassisi (2007) found a difference in comparing $\alpha$-enhanced and solar-scaled SSPs using $V, I$ and $K$ magnitudes could be attributed to the filters themselves. Coelho et al. (2007) found that for the majority of colour combinations the effect of $\alpha$ is in the same direction of metallicity, but in some (e.g. $(B-V))$ the effect is opposite to metallicity. For $(g-z)$ and $(g-K)$ the effect was unknown. We also tested the difference between GALEV $\alpha$-enhanced and solar scaled SSPs, produced using spectra from the BaSTI group with the $V, I$ and $K$ filters. The differences were slightly larger using the VIK filter

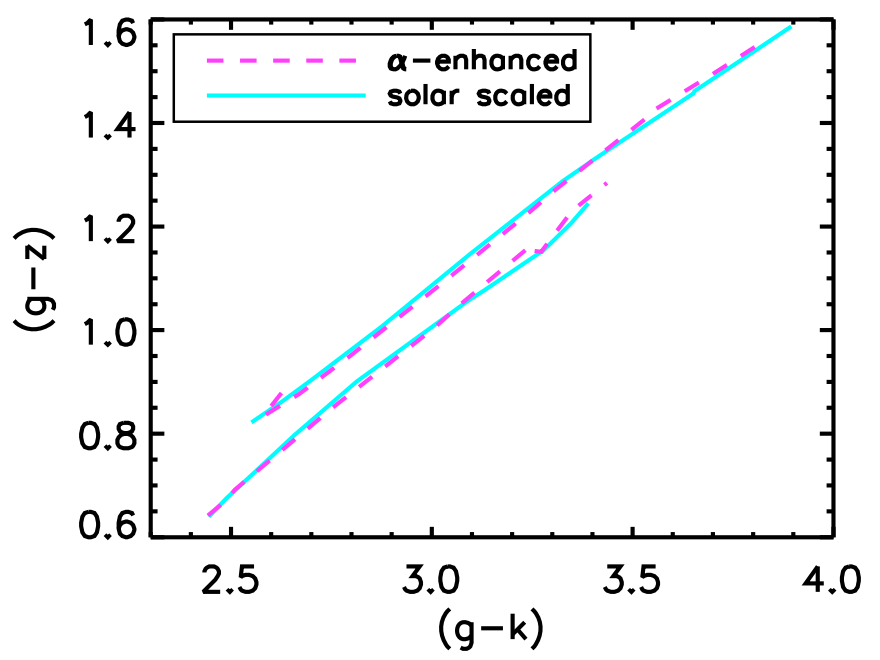

Fig. 15. A 2-colour diagram with $\alpha$-enhanced and solar scaled SSPs for the ages of 2 and 10 Gyr. The SSPs were produced in GALEV using spectra from the BaSTI group.

combinations instead of $g z K$, confirming the results of Salaris \& Cassisi (2007). We conclude therefore that through GALEV $\alpha$-enhanced SSPs using BaSTI spectra, $[\alpha / \mathrm{Fe}]$ variations would not contribute significantly to the scatter (i.e., $\sim 0.1-0.2 \mathrm{mag}$ ) in the 2 -colour diagrams using the $g z K$ filter combination, as done in this study.

\subsection{Horizontal-branch morphology}

In Fig. 16 the $(g-K)-(g-z)$ diagram for the joint NGC 4486 and NGC 4649 GC sample is shown with different models overplotted in different panels. Note the wavy feature the data presents around $(g-K) \sim 3.2$ and $(g-z) \sim 1.2$. A SpOT Teramo $14 \mathrm{Gyr}$ SSP with a detailed treatment of HB morphology (Raimondo et al. 2005) is overplotted in the top panel. Even though the track does not fit the redder part of the diagram well, it also presents the wavy feature seen in the data. Other SSP models that do not have a detailed prescription of the HB morphology do not show this behaviour (middle panel for Padova08 and bottom panel for Maraston 2005). As shown in Fig. 13, the scatter in the $\delta$ values is significantly reduced when going from a single linear relation between $(g-z)$ and $(g-K)$ to two linear relations. This separation into two linear relations simulates the effect of varying HB morphology in the 2-colour diagrams.

\subsection{Stochastic effects}

What would fluctuations in the number of bright stars do to the integrated colours of extragalactic GCs? We have generated synthetic integrated magnitudes and colours from models based on Padova isochrones (Marigo et al. 2008; Girardi et al. 2008) and a Monte Carlo method following Santos \& Frogel (1997) in order to assess the influence of stochastic effects on the integrated colours of the clusters. Padova isochrones for $g, z$, and $K$ were retrieved in a manner similar to the SSPs described in Sect. 3. A set of isochrones combining 18 ages $(2-14 \mathrm{Gyr}-\log t=$ 9.3 to 10.15 at 0.05 steps $)$ and 4 metallicities $(Z=0.00019$, $0.0006,0.0019,0.006)$ was employed. Integrated magnitudes and colours involving $g, z$ and $K$ bands were computed by distributing a prefixed number of stars on an isochrone of given age and metallicity according to a Salpeter initial mass function 
A. L. Chies-Santos et al.: Age distributions of GCs in early-type galaxies. II.

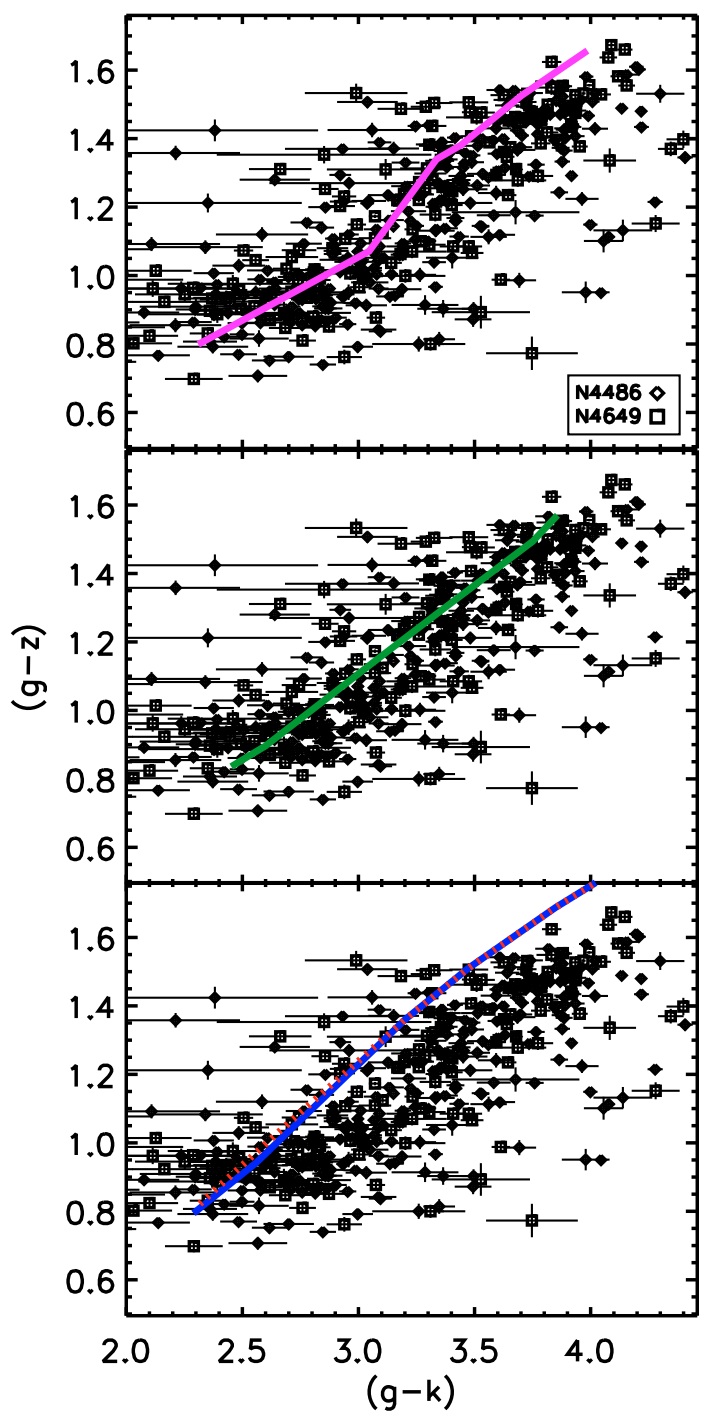

Fig. 16. $(g-K)$ vs. $(g-z)$ for GCs of NGC 4486 and NGC 4649, indicated by different symbols. Note the wavy feature the data presents around $(g-K) \sim 3.2$ and $(g-z) \sim 1.2$. The different panels have different SSP model over plotted: upper panel: SpOT-Teramo 14 Gyr SSP with a realistic treatment of horizontal branch morphology (Raimondo et al. 2005), middle panel: Padova08 and bottom panel: Maraston (2005).

(IMF). The use of a Kroupa mass function would not make a significant difference. The stochastic nature of the IMF was simulated by randomly sampling stellar masses between 0.08 and $120 M_{\odot}$ weighted on the IMF until the preset number of stars was reached. One hundred SSPs were simulated this way with their integrated fluxes spread reflecting the fluctuation in the number of bright stars. A GC with $g=23$ has $\sim 6 \times 10^{5} M_{\odot}$ at the distance of the Virgo cluster. This corresponds to $\sim 10^{6}$ stars, considering a Salpeter IMF. For simplicity, we have considered models with a fixed number of stars. We examine models of $10^{6}$ stars, since only the brightest GCs $(g<23)$ are in the sample.

In Fig. 17 the $(g-K)$ vs. $(g-z)$ synthetic diagrams are shown for $10^{6}$ stars. The upper panel shows that the scatter on the $(g-K)$ direction due to stochastic effects produced by a 2 Gyr SSP is slightly larger than that corresponding to the older, 14 Gyr one. The stochastic fluctuations are slightly higher for the younger SSPs compared to the older ones due to the fact that the main sequence turnoff point occurs at a higher mass for the $2 \mathrm{Gyr}$

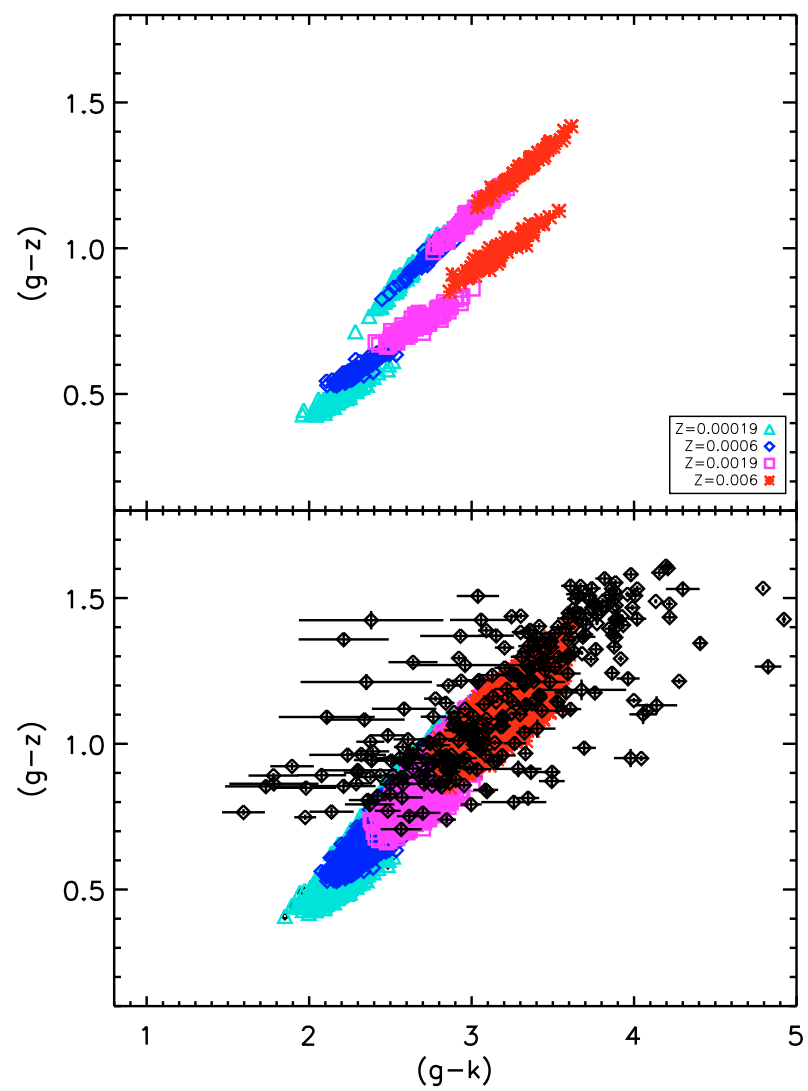

Fig. 17. The synthetic $(g-K)$ vs. $(g-z)$ diagrams for $10^{6}$ stars, for four different metallicities indicated by the different colours/symbols. Upper panel: for the ages of 2 and $14 \mathrm{Gyr}$. Bottom panel: for the age range of 2 to 14 Gyr and with the GCs of NGC 4486 over plotted.

SSP. This gives fewer RGB/AGB stars for a given total mass due to the combination of shorter stellar lifetimes and the IMF. The bottom panel shows that even for the combined age range of 2-14 Gyr, there is not a good match between the SSPs and the NGC 4486 GCs. The stochastic effects seem to be negligible as a source of colour spreading for such populous clusters. Most of the scatter due to stochastics is orthogonal to the direction of varying age, not contributing significantly to the scatter as plotted in Figs. 13 and 14.

\section{Discussion and implications for GC system assembly}

This study shows that the ages of the GC systems are correlated with the morphology of the host galaxy. Galaxies of later types are found to have GC systems with ages shifted towards younger relative values if compared to galaxies of earlier types. This finding can be crosschecked with host galaxy properties. Major star formation events should leave imprints both in the integrated light and in the GC system of a galaxy. Evidence of recent star formation in later-type galaxies is also found in integrated light studies. Shapiro et al. (2010) combine SAURON integrated field spectroscopy of early-type galaxies with Spitzer/IRAC imaging to investigate the presence of trace star formation in such systems. Connecting a detailed kinematic analysis given by the first dataset with direct probes of star formation from Spitzer, these authors find that star formation in early-type galaxies happens exclusively in fast rotating systems. In Fig. $18 \delta, \delta$ blue and $\delta_{\text {red }}$ are shown as function of $\lambda_{\mathrm{R}}$ for the the different galaxies from 


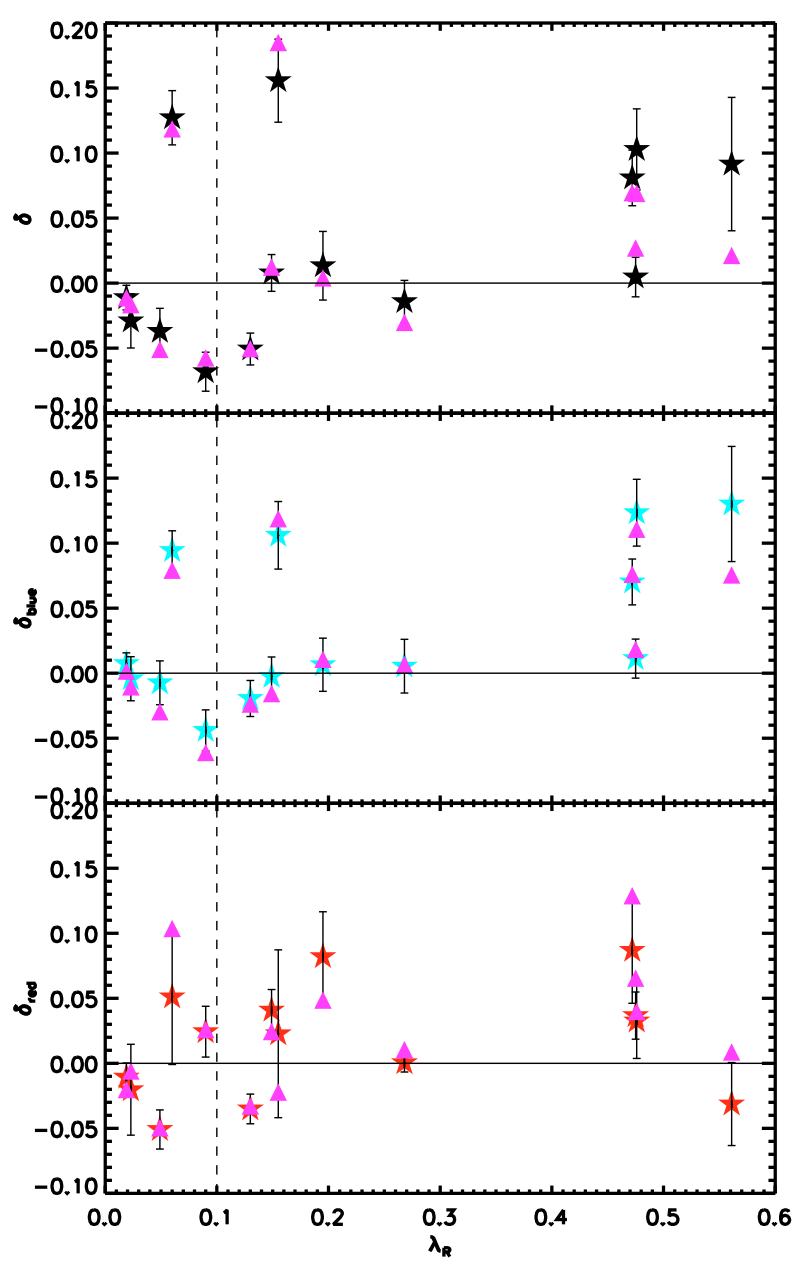

Fig. 18. $\delta, \delta_{\text {blue }}$ and $\delta_{\text {red }}$ Vs. $\lambda_{\mathrm{R}}$ parameter. The dashed vertical line corresponds to the division value between slow $\left(\lambda_{R}<0.1\right)$ and fast rotators $\left(\lambda_{R}>0.1\right)$. The solid line and the different symbols are as indicated in Fig. 12.

Emsellem et al. (2007) and Cappellari et al. (2007). This parameter is a proxy for the degree of rotation of the systems (Emsellem et al. 2007). Slow rotators have $\lambda_{\mathrm{R}}$ below 0.1 whereas fast rotators above 0.1 . Note that the GC systems consistent with younger ages in our picture, fall mostly among the fast rotators, with $\lambda_{\mathrm{R}}>0.1$, with a tendency for larger $\lambda_{\mathrm{R}}$ values $(>0.45)$ for $\delta$ and $\delta_{\text {blue. }}$. Slow rotators, on the other hand, seem to have a population of GCs consistent with $\delta$ and $\delta_{\text {blue }}<0$, implying old ages. This is suggestive of a more recent GC formation-connected to recent star formation in the host galaxy only occurring in fast rotating systems. The two outlying cases, with low $\lambda_{\mathrm{R}}$ values but high $\delta$ and $\delta_{\text {blue }}$ values are NGC $4406\left(\lambda_{\mathrm{R}}<0.1\right)$ and NGC 4382 $\left(\lambda_{\mathrm{R}}>0.1\right)$. There is evidence that the fast rotator NGC 4382 could have an artificially low measured $\lambda_{\mathrm{R}}$ in virtue of its significant inclination (see Emsellem et al. 2007).

Star formation occurring later in time in a galaxy is expected to come from metal-enriched gas. Therefore, one would presume that the metal-rich clusters would be driving this relation. However, this does not appear to be the case. The blue population appears to drive the relation between GC age and host galaxy morphological type. In this context, the blue GCs of the $\mathrm{E} 5 / \mathrm{SOs} / \mathrm{SAB}(0)$ are younger. This is surprising and contrary to the view that the metal-poor GCs formed (exclusively) at high redshift (Brodie \& Strader 2006). We have investigated whether this trend could be caused by systematics in the data such as seeing, aperture corrections, sky subtraction, etc., and conclude that this is not the case. There is, however a trend of anticorrelation with the number of clusters in a galaxy. However, this only reflects the fact that later type galaxies (S0's) have fewer clusters than earlier type ones (E0's). Furthermore, a trend between $\delta$ value and the brightness of the cluster is not present. Here, the direction of $\delta$ values shifts are interpreted as age shifts. However, given our limited understanding of SSP models and their necessary ingredients, there is still the possibility that this is not an age effect. It could be perhaps due to an unknown outcome of a certain group of stars in the clusters (e.g. HB, AGB).

A possibility is that the blue population drives the relation between GC age and host galaxy morphological type as a result of minor mergers. There would exist two possible ways for assembling these younger GCs. Dwarf galaxies have a more extended star formation history than galaxies of larger mass (Tolstoy et al. 2009) and contain almost exclusively metal-poor GCs (Forbes et al. 2000). One possibility is that these blue GCs formed in the dwarf galaxies prior to accretion (Côté et al. 1998). Another possibility is that the GCs were formed during the merger event from the gas reservoir of accreted dwarfs, presumably with metal-poor gas. Evidence for this latter possibility is found in Muratov \& Gnedin (2010). In this model for GC formation in the hierarchical framework of galaxy assembly, mergers of smaller hosts create exclusively blue clusters and mergers of more massive galaxies create both red and blue clusters. It remains uncertain, however, whether such effects would be expected to operate more prominently in S0-type galaxies compared to ellipticals. It is also unclear to what extent the age distributions of blue GCs in S0-type galaxies are directly related to the presence of recent star formation in the discs as revealed by SAURON. Unlike the blue GCs, the discs tend to be metal-rich, and spatially our blue GCs do not appear to be associated with the discs either.

In the Milky Way, there is substantial evidence that part of its GC system is associated with accreted dwarf galaxies. The age-metallicity relation study of Forbes \& Bridges (2010) for galactic GCs suggests that there are two tracks of objects in the Galaxy. One with constant old ages $\sim 13 \mathrm{Gyr}$, spanning a large metallicity range $-2.2<[\mathrm{Fe} / \mathrm{H}]<-0.6$, possibly formed in situ. The second track branches to younger ages and the objects appear clumped at a mean age value of $\sim 10.5$ Gyr and intermediate metallicity, $[\mathrm{Fe} / \mathrm{H}] \sim-1.3$. This latter track seems to be dominated by GCs associated with dwarf galaxies accreted by the Galaxy. A $\delta \sim 0.05-0.1$ corresponds in very rough terms to an age shift of $\sim 2-8$ Gyr. Such an age shift is therefore comparable to the difference in age between GCs formed in situ and GCs accreted from dwarf galaxies in the Milky Way. Moreover the $[\mathrm{Fe} / \mathrm{H}]$ value $(\sim-1.3)$ of this clump of objects, associated to dwarf galaxies, is closer to the metal-poor peak metallicity value than to the metal-rich one of GC systems of early-type galaxies. For example, the metal-poor peak of NGC 4406 is at $[\mathrm{Fe} / \mathrm{H}]=-1.23$, whereas the metal-rich one at $[\mathrm{Fe} / \mathrm{H}]=-0.56$ (Brodie \& Strader 2006, based on the empirical relation of Peng et al. 2006). Therefore if this younger clump of objects of the Galaxy was to be placed in NGC 4406, for example, it would belong to its metal-poor population. This is suggestive of younger GCs possibly belonging to the metal-poor population of a galaxy. In this context the age and metallicity of the blue population of clusters are related to the objects associated to dwarf galaxies.

There is also a trend of later type galaxies exhibiting a greater positive $\delta_{\text {red }}$ shift than earlier type ones (bottom panel of Fig. 12), although with much more scatter than for $\delta_{\text {blue }}$. The maximum 
shift occurs for E5 galaxies. Such a significant $\delta_{\text {red }}$ shift could be indicative of the host galaxy having experienced a major merger. From the Ashman \& Zepf (1992) scenario, formation of metal-rich GCs would occur during the merger of disc galaxies. Alternatively, it is tempting to link the large $\delta_{\text {red }}$ values to the amount of "disc" formation that happened in a particular galaxy (Kuntschner et al. 2006). For example, the fast rotators with large $\delta_{\text {red }}$ values, NGC 4660, NGC 4473 and NGC 4526 have a prominent $\mathrm{Mg} b$ enhanced, metal-enriched central disc-like structure (Kuntschner et al. 2006, 2010).

For all this to get beyond speculation, further investigation is required. Increasing the number of measured $\delta$ 's for different GC system would perhaps strengthen (or weaken) the relation between GC system age and morphological galaxy type. Spectroscopy of GCs in later early-type galaxies (S0's, perhaps E5's) might give it further confirmation. Moreover, cosmological simulations up to GC system formation and assembly could give further constraints on the nature of the mechanisms that shape the age distributions of GCs in present day early-type galaxies.

This study also shows that the age distribution of the GC system of NGC 4365 is not different from other similar type galaxies (e.g. NGC 4486 and NGC 4649). The optical colour distribution of this GC system is indeed different from others. This does not imply however, significant differences in the ages, but rather in the metallicity distributions.

\section{Summary and conclusions}

Using multi-band $g, z$ and the newly derived $K$-band photometry, we have compared differentially the GC systems in a sample of $14 \mathrm{E} / \mathrm{S} 0$ galaxies. We have also studied the effect of different parameters in various SSP models. The results are outlined below.

1. The most recent Padova SSPs for old ages show less of an offset with respect to the photometry than previously published models. However, a formal fit still yields intermediate ages.

2. The age distribution of the GC system of NGC 4365 appears similar to that of other large ellipticals in the sample. Although its colour distribution is different from other systems in $(g-z)$, this difference is orthogonal to the direction of decreasing/increasing age shift in the $(g-K)-(g-z)$ plane.

3. When the Padova08 SSPs integrated colours are used as SEDs the fits of the median ages of the blue and red subpopulations with AnalySED are found to be consistent with a single age, within one sigma. This makes them consistent with a great range of age values (from 1 to $14 \mathrm{Gyr}$ ), with the exception of some $\mathrm{S} 0$ galaxies.

4. A differential comparison without using SSP models is performed. This comparison is done in the direction of age shift, traced by the quantity $\delta$. A correlation between galaxy morphological type and the mean delta is found. Galaxies of earlier type host genuinely old GCs and galaxies of later type, younger on average clusters. The blue population appears to be driving this relation. The relation for the red population is not as clear as for the blue one.

5. The $(g-k)$ vs. $(g-z)$ parameter space is analysed and it is concluded that there is a non-linear feature around $(g-$ $k) \sim 3.2$ and $(g-z) \sim 1.2$. This feature is suggested to be due to the HB morphology. It is also present in SpOTTeramo SSPs which have a detailed modelling of this stellar evolution phase.
6. The influence of stochastic effects is investigated to address the spread seen in the 2-colour planes. It is concluded that the observational uncertainties are larger than the influence of stochastic effects for the cluster mass relevant to our study. In fact, the observational uncertainties can account for most of the observed scatter when considering blue and red clusters as separate populations.

7. We find that there is residual scatter in the delta parameter at bright magnitudes that cannot be accounted for by observational errors. This may indicate an intrinsic scatter in the GC ages of up to several Gyr.

Acknowledgements. We thank the referee for comments that improved the paper. We acknowledge Paula Coelho for interesting discussions. J.F.C.S.J. acknowledges the Brazilian institution FAPEMIG (grant APQ-00117/08). This research has made use of the NASA/IPAC Extragalactic Database (NED) which is operated by the Jet Propulsion Laboratory, California Institute of Technology, under contract with the National Aeronautics and Space Administration

\section{References}

Acosta Pulido, J. A., Ballesteros, E., Barreto, M., et al. 2003, ING News1., 7, 15 Anders, P., Bissantz, N., Fritze-v. Alvensleben, U., \& de Grijs, R. 2004, MNRAS, 347, 196

Ashman, K. M., \& Zepf, S. E. 1992, ApJ, 384, 50

Ashman, K. M., Bird, C. M., \& Zepf, S. E. 1994, AJ, 108, 2348

Brodie, J. P., \& Strader, J. 2006, ARA\&A, 44, 193

Cappellari, M., Emsellem, E., Bacon, R., et al. 2007, MNRAS, 379, 418

Cenarro, A. J., Beasley, M. A., Strader, J., Brodie, J. P., \& Forbes, D. A. 2007, AJ, 134, 391

Chies-Santos, A. L., Larsen, S. S., Wehner, E. M., et al. 2011, A\&A, 525, A19 (Paper I)

Coelho, P., Bruzual, G., Charlot, et al. 2007, MNRAS, 382, 498

Cohen, J. G., Blakeslee, J. P., \& Ryzhov, A. 1998, ApJ, 496, 808

Cohen, J. G., Blakeslee, J. P., \& Côté, 2003, ApJ, 592, 866

Côté, P., Marzke, R. O., \& West, M. J. 1998, ApJ, 501, 554

Elmegreen, B. G., \& Efremov, Y. N. 1997, ApJ, 480, 235

Emsellem, E., Cappellari, M., Krajnović, D., et al. 2007, MNRAS, 379, 401

Ferrarese, L., Côté, P., Jordán, A., et al. 2006, ApJS, 164, 334

Forbes, D. A., \& Bridges, T. 2010, MNRAS, 404, 1203

Forbes, D. A., Brodie, J. P., \& Huchra, J. 1997a, AJ, 113, 887

Forbes, D. A., Brodie, J. P., \& Grillmair, C. J. 1997b, AJ, 113, 1652

Forbes, D. A., Masters, K. L., Minniti, D., \& Barmby, P. 2000, A\&A, 358, 471

Girardi, L., Dalcanton, J., Williams, B., et al. (the ANGST/ANGRRR Collaboration) 2008, PASP, 120, 583

Goudfrooij, P., Mack, J., Kissler-Patig, M., Meylan, G., \& Minniti, D. 2001a, MNRAS, 322, 643

Goudfrooij, P., Alonso, M. V., Maraston, C., \& Minniti, D. 2001b, MNRAS, 328, 237

Hempel, M., Hilker, M., Kissler-Patig, M., et al. 2003, A\&A, 405, 487

Hempel, M., Zepf, S., Kundu, A., Geisler, D., \& Maccarone, T. J. 2007a, ApJ, 661,768

Hempel, M., Kissler-Patig, M., Puzia, T. H., \& Hilker, M. 2007b, A\&A, 463, 493 Kormendy, J., Fisher, D. B., Cornell, M. E., \& Bender, R. 2009, ApJS, 182, 216

Kotulla, R., Fritze, U., \& Anders, P. 2008, MNRAS, 387, 1149

Kuntschner, H. 2000, MNRAS, 315, 184

Kuntschner, H., Emsellem, E., Bacon, R., et al. 2006, MNRAS, 369, 497

Kuntschner, H., Emsellem, E., Bacon, R., et al. 2010, MNRAS, 408, 97

Larsen, S. S. 1999, A\&AS, 139, 393

Larsen, S. S., \& Brodie, J. P. 2000, AJ, 120, 2938

Larsen, S. S., Brodie, J. P., Huchra, J. P., Forbes, D. A., \& Grillmair, C. J. 2001, AJ, 121, 2974

Larsen, S. S., Brodie, J. P., \& Strader, J. 2005, A\&A, 443, 413

Lee, Y. W., Demarque, P., \& Zinn, R. 1994, ApJ, 423, 248

Manchado, A., Barreto, M., Acosta-Pulido, et al. 2004, SPIE, 5492, 1094

Maraston, C. 2005, MNRAS, 362, 799

Marigo, P., Girardi, L., Bressan, A., et al. 2008, A\&A, 482, 883

Muratov, A. L., \& Gnedin, O. Y. 2010, ApJ, 718, 1266

Peng, E. W., Jordán, A., Côté, P., et al. 2006, ApJ, 639, 95

Piotto, G., Bedin, L. R., Anderson, J., et al. 2007, ApJ, 661, 53

Puzia, T. H., Zepf, S. E., Kissler-Patig, M., et al. 2002, A\&A, 391, 453

Puzia, T. H., Kissler-Patig, M., Thomas, D., et al. 2004, A\&A, 415, 123 
A\&A 525, A20 (2011)

Puzia, T. H., Kissler-Patig, M., Thomas, D., et al. 2005, A\&A, 439, 997

White, S. D. M. 1978, MNRAS, 184, 185

Worthey, G. 1994, ApJS, 95, 107

Raimondo, G., Brocato, E., Cantiello, M., \& Capaccioli, M. 2005, AJ, 130, 2625

Salaris, M., \& Cassisi, S. 2007, A\&A, 461, 493

Santos, Jr., J. F. C., \& Frogel, J. A. 1997, ApJ, 479, 764

Shapiro, K. L., Falcón-Barroso, J., van de Ven, G., et al. 2010, MNRAS, 402, 2140

Sharina, M. E., Chandar, R., Puzia, T. H., Goudfrooij, P., \& Davoust, E. 2010 MNRAS, 405, 839
Springel, V., White, S. D. M., Jenkins, A., et al. 2005, Nature, 435, 629 Stetson, P. B. 1987, PASP, 99, 191

Strader,J., Brodie, J. P., Cenarro, A. J., Beasley, M. A., \& Forbes, D. A. 2005, AJ, 130, 1315

Strader, J., Beasley, M. A., \& Brodie, J. P. 2007, AJ, 133, 2015

Tolstoy, E., Hill, V., \& Tosi, M. 2009, ARA\&A, 47, 371

Thomas, D., Maraston, C., Bender, R., \& Mendes de Oliveira, C. 2005, ApJ, 621, 0673

Yamada, Y., Arimoto, N., Vazdekis, A., \& Peletier, R. F. 2006, ApJ, 637, 200 de Zeeuw, P. T., Bureau, M., Emsellem, E., et al. 2002, MNRAS, 329, 513 